\title{
Calibration and performance of the Galileo solid- state imaging system in Jupiter orbit
}

\author{
Kenneth P. Klaasen \\ H. Herbert Breneman \\ William F. Cunningham \\ James M. Kaufman \\ Jet Propulsion Laboratory \\ California Institute of Technology \\ 4800 Oak Grove Drive \\ Pasadena, California 91109-8099 \\ E-mail: kenneth.p.klaasen@jpl.nasa.gov \\ James E. Klemaszewski \\ Arizona State University \\ Department of Geology \\ Box 871404 \\ Tempe, Arizona 85287-1404 \\ Kari P. Magee \\ Sterling Software \\ P.O. Box 70908 \\ Pasadena, California 91117-7908
}

Alfred S. McEwen

University of Arizona

Lunar and Planetary Laboratory

Space Sciences Building

1629 E. University Boulevard

Tucson, Arizona 85721-0092

Helen B. Mortensen

Jet Propulsion Laboratory

California Institute of Technology

4800 Oak Grove Drive

Pasadena, California 91109-8099

\section{Robert T. Pappalardo}

Brown University

Department of Geological Sciences

Box 1846

Providence, Rhode Island 02912

\section{David A. Senske}

Sterling Software

P.O. Box 70908

Pasadena, California 91117-7908

Robert J. Sullivan

Cornell University

Center for Radiophysics and Space

Research

308 Space Sciences Building

Ithaca, New York 14853

Ashwin R. Vasavada

California Institute of Technology

Geological and Planetary Sciences

Mail Stop 170-25

Pasadena, California 91125
Abstract. The solid-state imaging subsystem (SSI) on the National Aeronautics and Space Administration's (NASA's) Galileo Jupiter orbiter spacecraft has successfully completed its 2-yr primary mission exploring the Jovian system. The SSI has remained in remarkably stable calibration during the 8-yr flight, and the quality of the returned images is exceptional. Absolute spectral radiometric calibration has been determined to 4 to $6 \%$ across its eight spectral filters. Software and calibration files are available to enable radiometric, geometric, modulation transfer function (MTF), and scattered light image calibration. The charge-coupled device (CCD) detector endured the harsh radiation environment at Jupiter without significant damage and exhibited transient image noise effects at about the expected levels. A lossy integer cosine transform (ICT) data compressor proved essential to achieving the SSI science objectives given the low data transmission rate available from Jupiter due to a communication antenna failure. The ICT compressor does introduce certain artifacts in the images that must be controlled to acceptable levels by judicious choice of compression control parameter settings. The SSI team's expertise in using the compressor improved throughout the orbital operations phase and, coupled with a strategy using multiple playback passes of the spacecraft tape recorder, resulted in the successful return of 1645 unique images of Jupiter and its satellites. () 1999 Society of Photo-Optical Instrumentation Engineers. [S0091-3286(99)02007-3]

Subject terms: charge-coupled device camera; calibration; digital imaging; remote sensing; space instrumentation; astronomy; data compression.

Paper 980345 received Sep. 8, 1998; revised manuscript received Dec. 14, 1998; accepted for publication Jan. 20, 1999. 


\section{Introduction}

On December 7, 1997, the Galileo spacecraft successfully completed its 2-yr nominal mission of 11 orbits around Jupiter. One element of the Galileo scientific payload is an electronic camera, designated the solid-state imaging subsystem (SSI). The primary scientific objectives of the imaging experiment were to investigate the chemical composition and physical state of the Jovian satellites and the structure and dynamics of the Jovian atmosphere. The SSI consists of a 1.5-m focal length, $f / 8.5$ Cassegrain telescope coupled with an $800 \times 800$-pixel charge-coupled device (CCD) detector yielding a field of view of $8 \mathrm{mrad}$. Spectral discrimination is provided by means of eight spectral filters, any one of which can be selected into the optical path using a filter changing mechanism (see Table 3 in Sec. 3.3 for the effective wavelengths of the filters).

Detailed descriptions of the SSI optical, mechanical, and electrical characteristics have been published previously. ${ }^{1,2}$ A previous publication documented the SSI inflight calibration and performance characteristics and how they have evolved during the 6 -yr cruise to Jupiter. ${ }^{3}$

In this paper, we describe the performance of the SSI during Jupiter orbital operations. The limited data rate available from the spacecraft at Jupiter due to failure of its primary high-gain antenna (HGA) to properly deploy made it impossible to perform a complete instrument calibration during the orbital mission. Only limited calibration data were acquired. The results of the SSI calibrations that were performed in orbit, including its response to the hostile energetic-particle radiation environment at Jupiter, are summarized. A review of the available software and procedures for calibrating SSI images is included. The engineering performance of the camera and of the other Galileo spacecraft subsystems that provide essential support to the camera operation are discussed. The operation and performance of the onboard data compression functions and the impact of image compression on scientific interpretation are reviewed. Finally, we describe aspects of the flight operations that affected the quantity and quality of the SSI data return.

\section{Spatial Resolution}

Only two attempts have been made to measure the system point spread function (PSF) of the camera in flight, both using imaging of selected photometric stars. The first used data acquired during Galileo's second Earth/Moon encounter (E/M-2) in December 1992, and the second attempt used data from the C9 orbit in July 1997 (Galileo orbits are denoted with the first initial of the targeted satellite, $\mathrm{E}=$ Europa, $\mathrm{G}=$ Ganymede, $\mathrm{C}=$ Callisto, followed by the number of the orbit about Jupiter). The results of the first analysis have been reported previously. ${ }^{3}$ Because the failure of the HGA resulted in severely curtailed data rates, the C9 data were acquired using $2 \times 6$ position multipleexposure on-chip mosaics (OCMs; the multiple exposures enable several images of a target that underfills the camera field of view to be acquired with a single frame readout using slight pointing changes between exposures to offset the images from one another ${ }^{3}$ ) with shutter events occurring every $82 / 3 \mathrm{~s}$. This resulted in an image residence time on the CCD of slightly more than $2 \mathrm{~min}$. All eight filters were used, with each OCM containing six star images from one filter and another six from a different filter. Because of the limited data rate available from Jupiter, the C9 star images had to serve double duty as both PSF and radiometric calibration sources. Relatively long exposures were used to yield high SNRs for radiometric accuracy at the possible expense of the best possible spatial resolution. All of the C9 star images were obtained at a Jupiter distance of $128 R_{\mathrm{J}}$ $\left(R_{\mathrm{J}}=\right.$ Jupiter's radius $\left.=71,398 \mathrm{~km}\right)$, where the radiation effects on the camera were relatively benign. The photometric stars used were $\beta$ Ari $\left(m_{v}=2.65\right.$, A5) and $\zeta$ Peg $\left(m_{v}\right.$ $=3.47, \mathrm{~B} 8)$. The images of $\beta$ Ari in the 889 -nm filter were lost due to a ground station outage.

The SSI line-spread function (LSF) was measured from the star images in a manner similar to that used on previous data. ${ }^{3,4}$ The analysis was limited to measuring the response only in the line (or vertical) direction. Measurements of the response in the sample (or horizontal) direction could not be made accurately because there is a deferred charge effect resulting from a charge trap in sample 170 of the CCD's horizontal register. ${ }^{3}$ This trap produces elevated wing responses at samples beyond sample 170 when there is a transition from high data numbers (DNs, which range from 0 to 255 for SSI data) to low DNs (such as occurs in these star images). The data were processed to remove known pixel-to-pixel variations, and small portions of the image surrounding each star were summed in the sample direction. The centroid in the line direction was determined, and the response relative to this centroid was measured. Typical LSF measurements using the C9 data are compared to those using the E/M-2 data in Fig. 1.

A comparison of the $\mathrm{C} 9$ data with the E/M-2 data shows that there were systematic increases in the widths and decreases in the peak response amplitudes of the LSFs for all filters. These changes could be due at least in part to the use of longer exposure times for the C9 images, thereby leading to a slight increase in image blurring from random scan platform jitter. Table 1 lists the LSF peak and full-widthat-half maximum (FWHM) values for each filter for both the E/M-2 and C9 analyses. The range of commanded exposure times of the images is also listed (for E/M-2, these apply only to the nonsaturated star images analyzed). The increased LSF widths for the C9 images are consistent with image smear levels in the range of 0.1 to 1.0 pixels. Given the exposure durations used in $\mathrm{C} 9$, these levels of smear would suggest a scan platform instability rate in the neighborhood of $1 \mathrm{pixel} / \mathrm{s}$ for the near-IR filters, but an instability rate closer to 10 pixels/s for the visible filters. The instability rate typically expected is about 5 pixels/s. Thus some additional degradation to the SSI spatial resolution in the visible wavelengths may have occurred since E/M-2 (conceivable causes might be propellant byproduct contamination or some defocusing in the SSI optics that affects primarily the shorter wavelengths), but the $C 9$ data are not very conclusive. Therefore, the C9 results place only upper limits on the widths of the SSI LSFs. The shorter exposures used for the E/M-2 and for the clear-filter C9 images seem to have kept smear to a nearly negligible level in those cases. The C9 clear-filter case suggests that the inherent system LSF of the camera has not changed substantially since the E/M-2 calibration. 


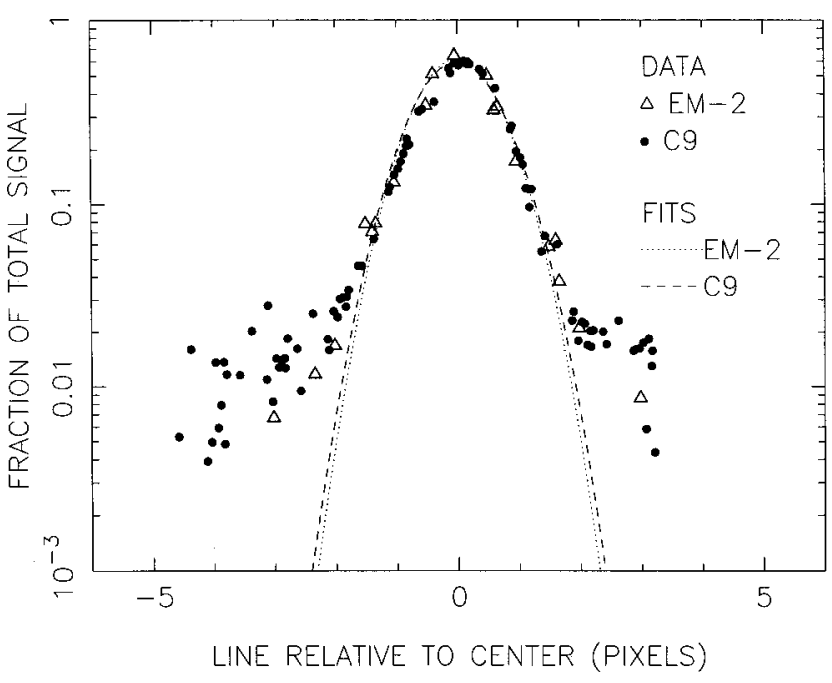

(a)

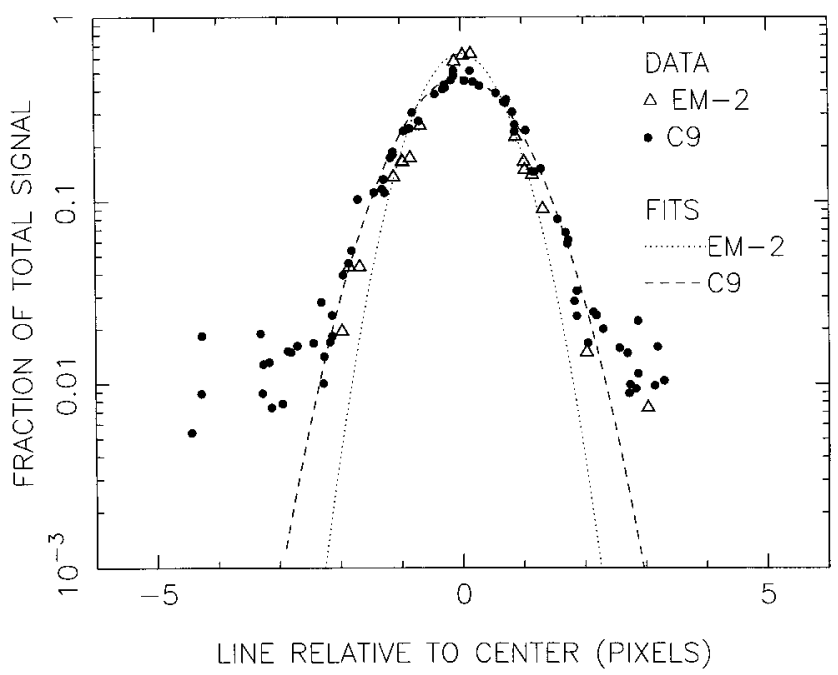

(b)

Fig. 1 SSI vertical LSF in the clear (a) and methane 1 (b) filters derived from star images acquired at the E/M-2 encounter (December 1992) and on orbit C9 about Jupiter (July 1997). The clear filter shows negligible change; the slight broadening of the methane 1 image is most likely due to increased image smear. The LSF peaks and widths for all the filters are listed in Table 1.

\section{Radiometric Calibration}

Only two limited SSI data sets could be returned during the orbital mission to address the task of calibrating radiometric performance. These sets comprised (1) a small set of zero-exposure frames in the newly implemented summation mode (one image in each of the four gain states) and (2) a more complete set of zero-exposure frames (covering all mode/gain combinations), flat-field images of the onboard photometric calibration target (PCT) using each of the eight spectral filters in both full-resolution and summation modes, and multiple images of two photometric standard stars using all eight filters. The downlink telemetry capability allocated for calibration was not sufficient to enable a recharacterization of the SSI shutter offset performance, detector linearity, signal gain coefficients, or system noise. These parameters are assumed to have remained constant since previous calibrations; there is no evidence that contradicts this assumption.

\subsection{Zero-Exposure Level}

The most important zero-exposure level data required for proper image calibration during orbital operations were those for the newly implemented $2 \times 2$-pixel summation mode designated "HIS", 3 This mode was defined during the cruise to Jupiter and implemented just before the start of SSI orbital operations. It had never been calibrated before; therefore, the zero-exposure levels were very uncertain. This mode became the primary imaging mode for observations of Jupiter. One high-priority Jupiter science objective is to perform radiative transfer inversions of the multispectral imaging data to reconstruct the vertical structure of the Jovian atmosphere. Thus proper radiometric calibrations in the HIS mode became of critical importance. As a result, a minimal zero-exposure data set consisting of one frame in each gain state in HIS was returned on the second orbit (designated G2). But because of the extremely limited data return capability, these images had to be returned highly compressed (Sec. 7). Thus, the high-spatialfrequency information was largely lost; only the lowfrequency levels were reliable.

HIS zero-exposure calibration files were constructed by extracting the high-frequency signatures from 2 1/3-s AI8 summation-mode zero-exposure images acquired at E/M-2 and combining those with the low-frequency offset levels

Table 1 Comparison of SSI LSF peaks and FWHM for the E/M-2 and C9 star image measurements.

\begin{tabular}{|c|c|c|c|c|c|c|c|}
\hline \multirow[b]{2}{*}{ Filter } & \multicolumn{3}{|c|}{$\mathrm{E} / \mathrm{M}-2$} & \multicolumn{3}{|c|}{ C9 } & \multirow{2}{*}{$\begin{array}{c}\text { E/M-2 to C9 } \\
\text { FWHM Increase } \\
\text { (pixels) }\end{array}$} \\
\hline & $\begin{array}{c}\text { Exposure Times } \\
(\mathrm{ms})\end{array}$ & $\begin{array}{c}\text { Peak Fraction of } \\
\text { Total Signal }\end{array}$ & $\begin{array}{l}\text { FWHM } \\
\text { (pixels) }\end{array}$ & $\begin{array}{c}\text { Exposure Times } \\
(\mathrm{ms})\end{array}$ & $\begin{array}{c}\text { Peak Fraction of } \\
\text { Total Signal }\end{array}$ & $\begin{array}{l}\text { FWHM } \\
\text { (pixels) }\end{array}$ & \\
\hline Violet & $4.16-25$ & 0.88 & 1.07 & $66.7-100$ & 0.45 & 2.08 & 1.01 \\
\hline Green & 4.16 & 0.71 & 1.33 & $33.3-133$ & 0.45 & 2.08 & 0.75 \\
\hline Clear & 4.16 & 0.68 & 1.39 & $12.5-25$ & 0.59 & 1.58 & 0.19 \\
\hline Red & 4.16 & 0.64 & 1.46 & 100 & 0.49 & 1.93 & 0.47 \\
\hline Methane 1 & 12.5 & 0.64 & 1.46 & $533-800$ & 0.47 & 1.99 & 0.53 \\
\hline Near IR & 4.16 & 0.60 & 1.57 & $267-800$ & 0.49 & 1.91 & 0.34 \\
\hline Methane 2 & 12.5 & 0.58 & 1.63 & 800 & 0.41 & 2.27 & 0.64 \\
\hline $1 \mu \mathrm{m}$ & 12.5 & 0.56 & 1.67 & $400-800$ & 0.45 & 2.08 & 0.41 \\
\hline
\end{tabular}


obtained from the G2 orbit. The mean zero-exposure DNs for the HIS-mode G2 images are:

gain state $1-2.99 \mathrm{DN}$

gain state $2-5.13 \mathrm{DN}$

gain state $3-9.11 \mathrm{DN}$

gain state $4-36.35 \mathrm{DN}$.

A full set of zero-exposure images was acquired on the ninth orbit (C9) in all modes and gain states. These images showed some high-frequency differences from the comparable AI8 frames acquired at E/M-2. These differences were due to changes in the locations of anomalous CCD dark spike pixels (Sec. 6.1), the signatures of transient energetic particle interactions, subtle changes to the $2400-\mathrm{Hz}$ coherent noise pattern from the spacecraft power supply, slight differences in columns 170 and 610 (locations of known charge traps in the CCD horizontal register), and small effects due to the use of lossy data compression (compression ratios ranging between 2:1 and 8:1 were used). Changes to the mean DN levels and the lowfrequency DN variations across the frame were minimal, measuring less than $1 \mathrm{DN}$ (average change of $0.2 \mathrm{DN}$ ) in all modes and gain states except gain state 4 (the highest gain setting) where the changes reached just less than $2 \mathrm{DN}$ (average change of $1 \mathrm{DN}$ ). Revised calibration files were constructed from the C9 images, and these are being used to calibrate all orbital images (Sec. 4).

\subsection{Absolute Sensitivity}

The images acquired of two photometric standard stars and the PCT on the C9 orbit enabled a check and refinement of the SSI absolute sensitivity calibration. The two stars selected, $\beta$ Ari $\left(m_{v}=2.65\right.$, A5) and $\zeta$ Peg $\left(m_{v}=3.47\right.$, B8) were also used during the E/M-2 calibration, so a direct comparison of the SSI response could be made. The absolute fluxes for these stars were obtained from ground-based observations $^{5-7}$ in the same manner as for the E/M-2 calibrations. ${ }^{3}$ Fluxes are estimated to be uncertain to about $3 \%$ in the middle wavelengths, about $5 \%$ below $465 \mathrm{~nm}$, and possibly as large as $15 \%$ in the range above $995 \mathrm{~nm}$. Six images of each star were obtained in each filter using the OCM technique. A ground tracking station outage resulted in loss of the 889-nm filter images of $\beta$ Ari. Single PCT images were obtained in each filter in both the fullresolution and summation modes.

SSI signal generation rates for the photometric standard stars observed in $\mathrm{C} 9$ were compared to those observed at the E/M-2 encounter as well as with the rates predicted by the final SSI spectral response model derived from cruise calibration data. ${ }^{3}$ The resulting C9 to E/M-2 response ratios are shown in Fig. 2. The average of all the ratios is 1.012 for the star images. The largest change observed was in the clear filter, which had an average response ratio of 1.118 relative to the E/M-2 and model predictions. Individual star signal measurements are uncertain to about $\pm 5 \%$; ratios of signals are then uncertain to about $\pm 7 \%$. There does not seem to be any reason to conclude that substantial changes occurred in the SSI absolute sensitivity between the E/M-2 encounter and the Jupiter orbital operations time frame nearly $5 \mathrm{yr}$ later based on the $\mathrm{C} 9$ star images.

SSI signal generation rates for the PCT on $\mathrm{C} 9$ were also compared to those observed at E/M-2 after adjusting for the

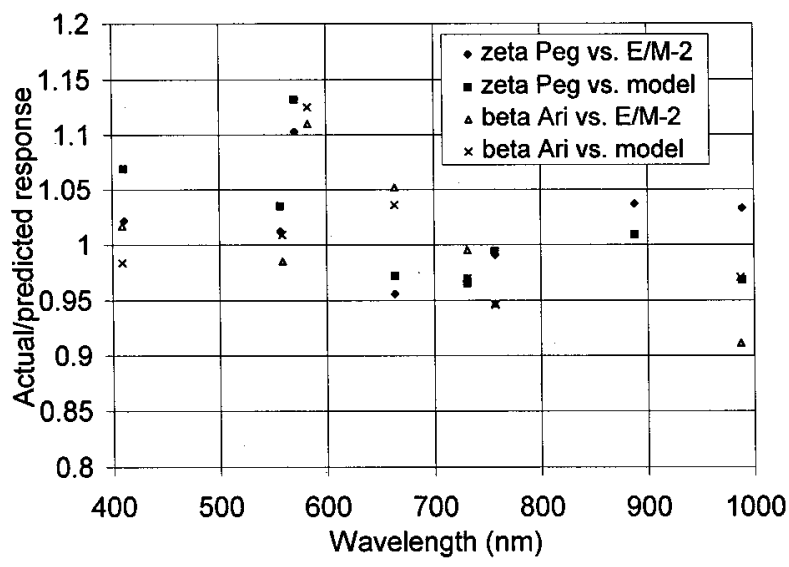

Fig. 2 Ratio of the actual SSI spectral response in each of its eight spectral filters to selected photometric standard stars measured during the Jupiter orbital mission to the response from similar observations made at the E/M-2 encounter and that predicted using the SSI spectral response model derived from interplanetary cruise calibration data.

change in PCT illumination with solar distance. The resulting $\mathrm{C} 9$ to $\mathrm{E} / \mathrm{M}-2$ ratios are plotted in Fig. 3. The C9 signal generation rates average about $95 \%$ of those at E/M-2 with a systematic trend of lower response at shorter wavelengths. The largest change is for the violet filter with a C9 to E/M-2 ratio of 0.895 . Since the star data indicate little change in the SSI spectral response, these data suggest that the PCT could have darkened and reddened slightly over the time interval between these calibrations, possibly due to contamination by thruster propellant byproducts.

Since the C9 SSI response data showed no obvious systematic differences from the E/M-2 performance above the measurement uncertainties, we conclude that the SSI absolute sensitivity in each filter has remained essentially unchanged throughout the mission. The C9 PCT and star response data were thus combined with the optics-cover-off

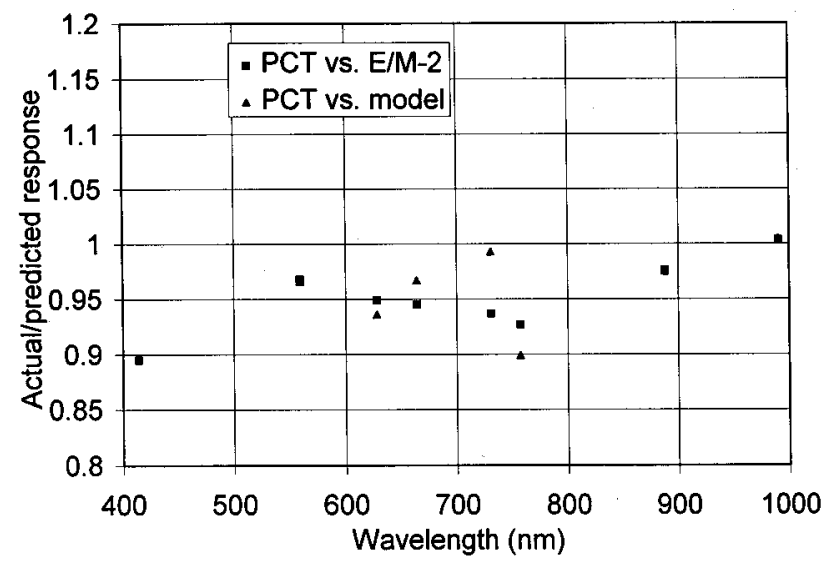

Fig. 3 Ratio of the actual SSI spectral response in each of its eight spectral filters to the onboard PCT measured during the Jupiter orbital mission to the response predicted from similar observations made at the E/M-2 encounter and that predicted using the SSI spectral response model derived from interplanetary cruise calibration data. 
Klaasen et al.: Calibration and performance of the Galileo ...

Table $2 \mathrm{SSI}$ absolute radiometric response in DN/

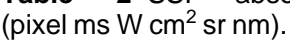

\begin{tabular}{lcccc}
\hline \hline Filter & Cruise & Orbit & Ratio & $\begin{array}{c}1-\sigma \text { Residual } \\
\text { (percent) }\end{array}$ \\
\hline Violet & $4.85 \times 10^{5}$ & $4.87 \times 10^{5}$ & 1.004 & 5.3 \\
Green & $2.05 \times 10^{6}$ & $2.04 \times 10^{6}$ & 0.998 & 4.2 \\
Clear & $1.71 \times 10^{7}$ & $1.71 \times 10^{7}$ & 1.001 & 5.2 \\
Red & $2.68 \times 10^{6}$ & $2.67 \times 10^{6}$ & 0.997 & 3.6 \\
Methane 1 & $2.67 \times 10^{5}$ & $2.64 \times 10^{5}$ & 0.990 & 3.6 \\
Near IR & $5.38 \times 10^{5}$ & $5.31 \times 10^{5}$ & 0.987 & 3.9 \\
Methane 2 & $1.46 \times 10^{5}$ & $1.45 \times 10^{5}$ & 0.993 & 3.7 \\
$1 \mu \mathrm{m}$ & $1.70 \times 10^{5}$ & $1.67 \times 10^{5}$ & 0.982 & 6.1 \\
\hline \hline
\end{tabular}

response data from interplanetary cruise to determine a refined calibration of the SSI inflight absolute spectral sensitivity using a least-squares solution. For this solution, the PCT spectral albedo was taken to equal that measured at the center of the PCT during prelaunch calibration for the light source 6 deg off axis; this spectrum provides a better match to the inflight measurements than the simple spectrally neutral 0.05 -albedo PCT assumed for the cruise calibration solution. ${ }^{3}$ The newly calibrated SSI response averaged over all pixels in units of DN per pixel per millisecond in response to a spectrally neutral scene radiance is listed in Table 2 for each filter in gain state 2 . The comparable values that resulted from the cruise-only calibration $^{3}$ are also listed along with the one-sigma residual modeling errors after the least-squares fit. The SSI sensitivity model has changed only very slightly as a result of including the C9 measurements. The largest change is less than $2 \%$, which is within the uncertainty of the previous calibration. The residual uncertainties are around $4 \%$ except at the wavelength extremes (and in the clear filter, which includes these extreme wavelengths) where the stellar radiances are less well known.

\subsection{Spectral Response Model}

The SSI spectral response model that was fit to the radiometric calibration data discussed includes specifications of the spectral transmission for the telescope optics and for each spectral filter, the spectral quantum efficiency of the CCD detector, and a conversion factor from signal electrons to DN (Ref. 3). The least-squares calibration solution adjusts each of these component specifications so as to yield a best-fit spectral response model for the instrument. The resulting best-fit SSI inflight spectral response model is plotted in Fig. 4. Table 3 lists the corresponding filter effective wavelengths and filter factors (ratio of clear filter response to that of the specified filter for the same exposure/gain setting) for both a spectrally neutral (gray) scene and a scene having the solar spectrum. This model was used to calculate the absolute radiometric conversion factors used in the SSI calibration software (Sec. 4).

\subsection{Individual Pixel Response}

The PCT flat-field images acquired on C9 enabled an assessment to be made of any changes that might have occurred in the relative response from pixel to pixel across the

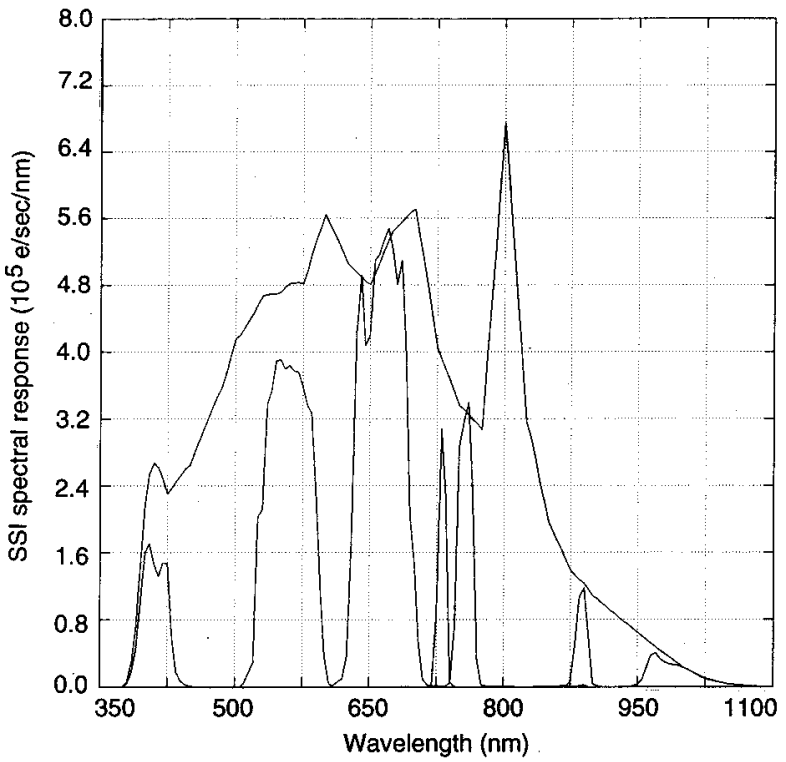

Fig. 4 Best-fit modeled SSI spectral response to a spectrally uniform gray radiance source of $1 / \pi \mathrm{W} / \mathrm{m}^{2} \mathrm{srnm}$ based on simultaneously matching calibration data acquired during cruise and in Jupiter orbit.

CCD array. Pixel-to-pixel response differences result from such things as CCD fixed pattern response differences, lowfull-well pixels, dust speck shadows, and corner vignetting. ${ }^{2,5,8}$ The degree to which such response differences may have changed between E/M-2 and C9 was evaluated by applying the radiometric calibration files derived from the E/M-2 images to the C9 images. Any departures from a spatially uniform output indicate a change that has taken place.

Other than known transient effects due to radiation interactions and time-variable dark spikes (Sec. 6), the only changes detected were a slight shift in the position of the new dusk speck shadow that first appeared ${ }^{3}$ in flight at E/M-2 and some small variations in the low-frequency shading across the frame. These differences introduce contrast at about $3 \%$ of the mean signal level. Revised flat-field calibration files have been generated incorporating these changes for use on images acquired in Jupiter orbit (Sec. 4).

Table 3 Effective wavelengths and filter factors.

\begin{tabular}{lccccc}
\hline & \multicolumn{2}{c}{ Gray Radiance } & & \multicolumn{2}{c}{ Solar Radiance } \\
\cline { 2 - 3 } \cline { 5 - 6 } Filter & $\lambda_{\text {eff }}$ & Filter Factor & & $\lambda_{\text {eff }}$ & Filter Factor \\
\hline Violet & 413.0 & 35.20 & & 413.7 & 34.28 \\
Green & 559.4 & 8.374 & & 559.0 & 6.926 \\
Clear & 651.6 & 1.0 & & 624.9 & 1.0 \\
Red & 664.6 & 6.405 & & 663.6 & 6.351 \\
Methane 1 & 731.2 & 64.82 & & 731.1 & 75.01 \\
Near IR & 756.9 & 32.27 & & 756.8 & 39.52 \\
Methane 2 & 887.6 & 118.2 & & 887.6 & 186.9 \\
1 $\mu$ m & 991.3 & 102.5 & & 989.7 & 202.3 \\
\hline \hline
\end{tabular}




\section{Calibration Software and Procedures}

Only two software packages exist (that we are aware of) that are capable of applying the basic radiometric and geometric calibrations to "raw" SSI images: VICAR [from the Jet Propulsion Laboratory (JPL) in Pasadena, California] and ISIS [from the U.S. Geological Survey (USGS) in Flagstaff, Arizona]. Calibration procedures via each package are described next.

\subsection{VICAR}

The SSI data can be processed using the VICAR image processing software set available from the JPL Multimission Image Processing Laboratory. Specialized modules or procedures specific to SSI data have been developed for radiometric calibration, modulation transfer function (MTF) correction, scattered light correction, unmosaicking OCMs, and fixing column blemishes (Sec. 6.1) identified in the SSI camera.

Radiometrically calibrating Galileo SSI images is a fivestep process involving several programs and procedures. The first step is performed as an option on integer cosine transform (ICT)-compressed images only (Sec. 7). The VICAR program ICTFIX is used to smooth artifacts that may be introduced in the image along the edges of the 8 $\times 8$-pixel boxes used in the ICT compression process.

The second step, using the VICAR program ADESPIKE, is also optional. ADESPIKE will detect and remove single-pixel errors (spikes) such as radiation noise events (Sec. 6.2). A pixel is determined to be in error if it differs from its adjacent neighbors by more than a userspecified threshold. Pixel values found to be in error are replaced by the average value of the adjacent pixels. The despiking process may be performed more than once on a given image.

The third step is to use the VICAR program GALSOS to convert each raw DN value to absolute units of reflectance or radiance. GALSOS will also remove camera blemishes and the zero-exposure background level, compute raw image entropy, and encode the locations of invalid pixels as bad-data records. Applying GALSOS is the only essential step in the radiometric calibration process. GALSOS uses a database to determine which calibration files to use for any given image, although the user can specify these files directly. More information regarding calibration files can be found at the URL http://rushmore.jpl.nasa.gov/ dnj/ GllDemo/Intro/Gll_Radiometry.html.

The fourth step is to use the VICAR program GLLFILLIN as an option to fill in any data gaps such as missing lines or partial lines caused by transmission outages. GLLFILLIN also fills in lines truncated prior to transmission by the SSI's data compressor. The fill performed is an interpolation between good lines. The interpolation is $1-\mathrm{D}$ and is always in the vertical direction. The maximum number of adjacent missing lines over which interpolation is to be done can be limited to a specified value.

The fifth step is accomplished using a procedure designed as an option to remove known column blemishes. The procedure is called GLLHOTPIX.PDF. The column blemishes are specified orbit by orbit since they vary with time, and a horizontal interpolation across good pixels sur- rounding the column blemish is performed to cosmetically remove it.

Geometric correction of Galileo images is typically performed only at the time of data reprojection into other map coordinate systems. The correction is so small in image space that the correction is not required until reprojection of the data is performed. Data reprojection and geometric correction are done with the VICAR program MAP3.

An MTF correction can be performed to sharpen edges and restore their original frequency distribution. The MTF model is a linear Wiener convolution kernel performed in the spatial domain in which the power spectrum SNR is approximated by a constant. A procedure called MTFCORR.PDF facilitates this operation. Ground calibration imagery of linear edges was used to extract the camera MTF in both sample and line directions.

Scattered light caused by internal reflections within the camera baffling was a noticeable problem in some SSI images. It is modeled as an isoplanatic PSF with very broad wings. ${ }^{3,4}$ The resulting corrective Wiener kernel is sufficiently large that the image deconvolution is performed in the Fourier domain. To correct for scattered light, a collection of programs is used. The VICAR program GLLPSF is used to generate a Fourier transform of the point-spreadplus-scattered-light function for the given filter. The VICAR program FFT22 is then used on the output of GLLPSF to convert it to a 2-D optical transfer function (OTF). The input image to be corrected is converted to a 2-D fast Fourier transform (FFT) with the program FFT22. The image FFT and the PSF OTF then have the Wiener noise additive restoration model applied on a point-bypoint basis with the VICAR program WIENER. The output from the WIENER program is then converted back to an image with the program FFT22.

OCMs require the extraction of each shuttered event into its own individual image, with the correct ancillary information updated in its VICAR label. The program UNMOSAIC is used to identify the areas for extraction from each image that correspond most closely to each given shutter event and to assign them their corresponding spacecraft clock times. The program CATLABEL is used to update the VICAR label information.

VICAR can be obtained by sending e-mail to Danika.Jensen@jpl.nasa.gov. More information on VICAR can also be obtained at the URL http:// rushmore.jpl.nasa.gov/vicar.html.

\section{$4.2 I S I S$}

An alternative to VICAR is the ISIS (Integrated Software for Imagers and Spectrometers) software package developed and maintained by USGS. ISIS is available as publicdomain software via http://wwwflag.wr.usgs.gov/isis-bin/ isis.cgi and will run on a variety of computer platforms. The main strengths of ISIS are map reprojection routines, methods to remove random noise, the ability to deal with multiband image cubes as well as single-filter images with ease, and its integration with the commercial program IDL (Interactive Data Language) for graphical routines such as match point acquisition. However, ISIS lacks the equivalent of VICAR procedures ICTFIX and the scattered light 
corrections. There are 'isis2vicar' and 'vicar2isis' programs in both ISIS and VICAR that enable image conversions for processing in both packages.

The steps involved in processing a raw SSI image into a radiometrically calibrated image using ISIS are described next.

1. Raw images are converted either from VICAR or Planetary Data System (PDS) format (on CD-ROM) into ISIS cubes using 'vicar2isis' or 'pds2isis'. These programs retain the original image header and add keywords used by ISIS in the process of reformatting the labels.

2. Galileo SPICE ancillary information is then added to the image header using the program 'naiflab'. This program takes the image shutter event time and uses the SPICE files to determine the vectors describing the pointing of the camera and the positions of the spacecraft, target body, and sun.

3. The image is radiometrically calibrated with 'ssical'. This program uses the appropriate calibration files to scale the image from raw DN values into units of reflectance or radiance. This program performs the same function as the VICAR program GALSOS, and tests have shown that the results of the two programs are identical to within the roundoff errors.

4. Removal of noise hits and other image artifacts using 'boxfilter'. For noise removal, the STDZ filter option is run first, which can be set to null out any pixels that are a user-defined number of standard deviations above (and/or below) the average of the pixels surrounding. ${ }^{9}$ This process enables noise removal based on relative, not absolute, pixel brightness, and does a better job than the VICAR program ADESPIKE. The filter can be run several times in severe cases. Any obvious artifacts that are not removed by the STDZ filter, such as column blemishes, can be removed (set to null) by running the "MD" option in 'qview'. The nulls are then filled in (interpolated) using the LPFZ option of 'boxfilter'.

5. If ICT compression artifacts or scattered light must be reduced, images must be converted to VICAR format and the ICTFIX and/or the described scattered light corrections applied using VICAR.

\section{Engineering Performance}

In addition to the remarkably stable SSI scientific calibration, the SSI and the Galileo spacecraft performed extremely well throughout the nominal mission at Jupiter from an engineering standpoint. Only a few minor anomalies were experienced, and workarounds to these were rapidly defined and implemented. These problems and their effects on SSI images are discussed next.

\subsection{Camera Operation}

The first SSI anomaly experienced involved its internal block-adaptive rate-controlled (BARC) data compressor. ${ }^{10}$ This compressor is implemented in hardware and has two selectable operating modes. These modes differ in how they meet the constraint that each compressed SSI image line must be returned using a fixed number of bits $(2592$ bits, or an average of 3.24 bits/pixel over the SSI's 800 pixel lines versus 8 bits/pixel for uncompressed data). In the information-preserving mode, all transmitted pixels can have their original DN value reconstructed without error; however, if a line contains too much information to be completely encoded within the 2592-bit limit, i.e., the scene being imaged has too much entropy by virtue of the adjacent pixel DN differences having a wide variety of values, the number of pixels returned will be truncated to only the first $n$ that can be encoded within the bit allocation. In the rate-controlled mode, on the other hand, the compressor attempts to return all pixels in a line but with some resulting error in the DN reconstruction if the entropy is too high. In this mode, the compression algorithm reduces the initial digitized image entropy if necessary by truncating up to three of the least-significant bits (LSBs) from each pixel. This LSB truncation is performed sequentially, one LSB at a time over blocks of 64 adjacent pixels. The 64-pixel block with the highest entropy is selected for each successive LSB truncation up to the limit of 3 truncated LSBs per block. If the line still has too much entropy to fit within the 2592-bit limit even after every 64-pixel block has had the full 3 LSBs truncated, then pixels are dropped off the end of the line until the constraint is met.

The observed anomaly involved image line truncation occurring in the rate-controlled mode for high-entropy scenes prior to having all $3 \mathrm{LSBs}$ truncated from every block in the line. This excessive line truncation was seen in high-resolution Ganymede images from the first satellite encounter of the mission (G1). A subsequent examination of SSI BARC images acquired during interplanetary cruise and on the ground using the spacecraft testbed showed that they exhibited similar characteristics but with much less line truncation (because the scene entropies were not nearly as high). A detailed analysis of the BARC compression algorithm revealed that the algorithm's logic for estimating the number of LSB truncations required for a line to be encoded within the 2592-bit allocation was not consistent with the actual coder implementation. The image activity estimator included an implicit assumption that six coding operators are available within the BARC compressor; however, the actual coder implementation has only five. As a result, the number of LSB truncations required within a line is underestimated, and the coder runs out of bits early. This flaw reduces the value of the rate-controlled mode of BARC compression relative to that of the informationpreserving mode; hence, the rate-controlled mode was not selected for use as frequently as originally planned during the orbital mission.

The second SSI anomaly involved a difference between actual and commanded exposure times. During the G1 encounter, the signal levels generated in certain Europa pictures using a commanded exposure time of $81 / 3 \mathrm{~ms}$ were nearly the same as those generated in pictures of similar scenes using commanded exposure times of $41 / 6 \mathrm{~ms}$. In addition, similar signal levels were observed in images acquired with commanded exposure times of $121 / 2$ and 16 $2 / 3 \mathrm{~ms}$. This anomaly was investigated and determined to have been introduced inadvertently during the inflight modifications to the SSI flight software for orbital operations. One command in the original flight software was eliminated to help free up processing time required to ex- 
Klaasen et al.: Calibration and performance of the Galileo ...

Table 4 OCMs with scan platform slewing unintentionally commanded during long exposures.

\begin{tabular}{lllcc}
\hline \hline Orbit & Frame ID & \multicolumn{1}{c}{ Observation Type } & $\begin{array}{c}\text { Detectable } \\
\text { Streak } \\
\text { Expected? }\end{array}$ & $\begin{array}{c}\text { Streak } \\
\text { Visible? }\end{array}$ \\
\hline G1 & 3500297.45 & lo eclipse; clear & $\mathrm{Y}$ & $\mathrm{N}$ \\
G7 & 3895222.00 & Europa eclipse: clear & $\mathrm{Y}$ & $\mathrm{Y}$ \\
G7 & 3896083.68 & lo eclipse: clear/green/red/violet & $\mathrm{N}$ & $\mathrm{N}$ \\
C9 & 40170480.0 & lo eclipse: $1-\mu \mathrm{m} / \mathrm{clear}$ & $\mathrm{N}$ & $\mathrm{N}$ \\
C9 & 4019578.00 & lo eclipse: $1-\mu \mathrm{m} / \mathrm{clear}$ & $\mathrm{Y}$ & $\mathrm{Y}$ \\
\hline \hline
\end{tabular}

ecute the new flight software for orbital operations. This modification inadvertently causes the shutter to close $41 / 6$ ms early for all but three commanded exposure times. This anomaly, once understood, had little impact on the SSI investigation. Two of the shorter SSI exposure commands $(8$ $1 / 3$ and $121 / 2 \mathrm{~ms}$ ) became unavailable (they are effectively shortened so that they duplicate two of the three unaffected exposure commands). The effects on the other available exposure commands is quite small since those commands are all at least $25 \mathrm{~ms}$ long. It was determined that patching the SSI flight software to fix the problem was impractical. Therefore, it was decided to leave the SSI flight software as is. The SSI planning software was modified to take into account the $41 / 6-\mathrm{ms}$ exposure reductions in modeling the predicted SSI response and selecting optimum exposure commands. The SSI radiometric calibration software also was modified to reflect our new understanding of the actual exposure times achieved (Sec. 4).

The primary purpose of the SSI flight software modifications for Jupiter orbital operations was to implement several new capabilities and operating modes to enhance imaging science return for the reduced data rates available over the spacecraft low-gain antenna (the primary HGA failed to deploy properly early in the cruise phase). These new capabilities included the new $2 \times 2$-pixel HIS summation mode, two new partial-frame readout modes, and the multiple-exposure "on-chip mosaicking" capability. ${ }^{3}$ These modes all worked as expected and proved extremely beneficial in maximizing the value of the SSI investigation.

During the Jupiter orbital mission, the SSI executed 2455 shutter actuations and 1552 filter wheel steps, bringing the totals for the lifetime of the instrument to 54,340 shutter actuations and 24,955 filter wheel steps. The estimated life cycles for these mechanisms are 180,000 and 300,000 , respectively. Total power-on time has reached $31,355 \mathrm{~h}$, and the power has been cycled on/off 147 times.

\subsection{Spacecraft and Sequencing Operation}

With the failure of the HGA to properly deploy, the capability to return SSI data in real time from the spacecraft was lost. Therefore all SSI data had to be recorded initially on the spacecraft's tape recorder for later playback at a low data rate. On a few rare occasions, one or more SSI image lines were lost within the spacecraft's onboard data system. In orbit $\mathrm{C} 3$, two images were noted to contain discontinuities related to missing lines that were not recorded as the CCD was read out. Similar discontinuities were noted subsequently in images obtained during orbits E4, E6 and C9. During the prime mission, seven images were found to con- tain instances of such line drop-outs. Typically, missing lines occur once within an image and involve 2 to 6 lines. Only one image obtained in C9 has multiple line drop-outs within a frame. There does not appear to be any correlation between instances of line drop-outs and image mode, line number, or distance from Jupiter. Only one (possibly two) instances of missing lines correspond to known or predicted "tape dings," i.e., potentially damaged regions of tape caused by prior tape-sticking events ${ }^{3}$ that may interfere with proper recording. Images with missing lines detected are edited on the ground so as to position all image lines in their proper locations, leaving gaps for the missing lines. Causes for these line drop-outs are still under investigation.

During the primary mission, several incidents occurred in which the SSI shutter was unintentionally open while the scan platform was slewing between the mosaic positions of an OCM. This only occurred in OCMs containing long $(\geqslant 6.4 \mathrm{~s})$ exposures, which were used for imaging Io and Europa in eclipse. In some cases, the anomaly was apparent in the image as streaks connecting bright scene features (stars or hot spots on the surface of Io) in different OCM images in the frame.

Close examination of the command sequence for all such instances showed that the scan platform had been erroneously commanded to slew to the next mosaic position while the shutter was still open for the long exposure intended to be completed at the previous mosaic position. There was no evidence that either the scan platform or the SSI did not perform as commanded. The result of the premature slewing was that the effective exposure times of some images were significantly shorter than what was commanded. In cases where the scan platform arrived at the next position with the shutter still open, effective exposure times longer than commanded could result, and signals from exposures in different filters could be mixed in the same image.

This problem was first detected and diagnosed in an Io eclipse image during orbit C9. In response to this, similar designs from earlier orbits were rechecked in hindsight, and a few were found to have the same design flaw. In some cases, the command sequence was found to have slew timing errors, although no streaks were visually apparent in the image. This could be accounted for by low signal levels, high noise, or sluggish initiation of the scan platform slew for those particular images. For subsequent orbits, longexposure OCM designs were handled more carefully during the sequence development process, and there were no further occurrences. Table 4 lists the frames affected by this anomaly. 
During the primary mission, the stability of the Galileo scan platform (on which the SSI is mounted and which is used to point the remote sensing instruments at their desired targets) was exceptional, providing high-quality data with a minimum of image smear. Only a few cases have been identified in which substantial smearing occurred. These fall into four categories: (1) a single case interpreted to be associated with a slower than usual commanded slewto-target rate; (2) OCMs in which there was most likely residual scan platform settling motion between successive positions of the mosaic; (3) OCMs in which the scan platform was commanded to move prior to the shutter being closed (see earlier) and (4) long exposures, greater than 800 ms, which show motion due to scan platform "jitter." The worst case of image smear occurred during the G1 orbit and resulted in the first frame of a $1 \times 4$-frame mosaic being rendered unusable for scientific analysis. As part of a cooperative observation between SSI and the photopolarimeter radiometer (PPR) instrument, a relatively slow scan platform slewing rate was commanded so that PPR could acquire data during the SSI slew to target. Engineering data for the period over which this observation occurred is sparse, providing little insight into the scan platform activity. It is, however, suspected that the commanded slew rate was not sufficient for the platform to reach its intended target in the allocated time, thus resulting in instrument motion while the SSI shutter was open.

Scan platform pointing during the nominal mission generally performed as predicted. Calibration of the scan platform during cruise indicated that, except for cases using extreme cone angles (the pointing angle relative to the spacecraft's spin axis), a pointing uncertainty of $<0.5 \mathrm{mrad}$ (50 SSI pixels) could be expected. Observed pointing errors in excess of this level fall into five cases: (1) observations that occurred near the spacecraft cone pole [within $15 \mathrm{deg}$ of the cone pole, compensation in the platform pointing for spacecraft nutation and wobble is disabled in the clock direction (i.e., about the spacecraft's spin axis), which can contribute to a higher pointing uncertainty relative to data obtained at lower cone angles], (2) observations in which there were large target-body ephemeris uncertainties, (3) changes in pointing due to differences between the predicted and actual spacecraft delivery point at a satellite closest approach, (4) spacecraft star scanner bright-body avoidance and an attitude-control system gyro drift anomaly, and (5) activities for which there is insufficient engineering data to provide a firm conclusion as to the cause of the pointing problem. Each of these cases is discussed in turn.

Evidence of atypical pointing error was first observed during the Ganymede closest approach observations on the first orbit (G1). These data were acquired at cone angles between 158 and $164 \mathrm{deg}$ and used the overtravel method of targeting (linear scan platform motion across the cone pole coupled with moderate arc motion in clock as opposed to moving in excess of $180 \mathrm{deg}$ in clock). Images acquired during this period showed displacements from their predicted surface locations of up to $2.4 \mathrm{mrad}$.

On several occasions, observations of the inner small satellites (Metis, Adrastea, Amalthea, and Thebe) failed to capture the disk of the target body within the field of view. The identification of large satellite ephemeris uncertainties was found to contribute significantly to this problem. During the seventh orbit (G7), a set of optical navigation images was obtained to better determine the ephemeris for Adrastea. Analysis of these images has not conclusively identified the intended target. This may be due either to (1) the failure to capture the body within the area of the CCD that was played back or (2) generating insufficient signal from the satellite relative to that predicted for the chosen exposure.

Differences between the actual and predicted locations of image footprints can also result from dispersions in the spacecraft delivery point for a given satellite encounter. The general navigation procedure was to perform an orbit trim maneuver (OTM) 3 days prior to the scheduled satellite closest approach to place the spacecraft on its proper trajectory. In some cases, the predicted arrival point at E-3 days differed only slightly from the desired target point, and this maneuver was deemed not necessary. This occasionally resulted in pointing errors that exceeded the usual 0.5-mrad limit. Specific examples in which this occurred include a nontargeted G2 global-scale Callisto mosaic as well as some E6 closest-approach images, in which the frames were systematically shifted to the south of their intended locations by $\sim 2.4 \mathrm{mrad}$.

Anomalous spacecraft pointing that resulted in the loss of three image frames (including observations of Io, Adrastea and Metis) occurred during the inbound part of the G7 observation sequence. During the satellite encounter periods throughout the orbital tour, it was often necessary to disable the star scanner during a portion of each spacecraft spin period to avoid looking at bright bodies such as Jupiter and its satellites. For the G7 orbit, the exclusion area was specified with a boundary very close to the location of one of the stars needed for attitude reference. Although the selection of this sector was successfully simulated on the spacecraft testbed, it failed to work properly in flight. As a result, only one of the two selected stars was successfully detected, and the star reference algorithm failed, causing the spacecraft to automatically switch to gyros for attitude reference. Because the gyros have a small amount of inherent drift, the attitude reference information began to depart from the real spacecraft attitude. Without updated star reference data, the dispersion between the actual attitude and that derived from the gyros increased with time. Before the initiation of playback, it was determined that observations taken during the period that the spacecraft operated on gyros alone failed to acquire their desired targets. As a result, no attempt was made to return these data. Fortunately, just prior to Ganymede closest approach, the attitude reference was updated, and the remainder of the sequence executed as expected.

During the $\mathrm{C} 10$ and E11 orbits, significant pointing offsets were detected for sets of global-scale Europa and Io observations. In several cases, where long periods of time were required to shutter and record the data, it was observed that the pointing error would decrease with time. This behavior is consistent with anomalous spacecraft attitude control gyro drift activity, which was again observed and characterized during the first Europa encounter (E12) of the extended Galileo Europa mission (GEM).

Although in most cases it has been possible to identify the cause of disparities between the predicted and actual 
scan platform pointing, we have not always been able to do so. The G1 case mentioned earlier is one example of unexplained errors. In addition, during the sixth orbit (E6), substantial frame offset was observed for a global-scale $1 \times 2$-frame mosaic of the southern high latitudes of Europa. Due to the coarse sampling of engineering data, it has not been possible to determine the exact scan platform activity during the time over which this observation was performed. The cause of this pointing anomaly remains unknown.

\section{Detector Performance}

\subsection{CCD Dark Spikes}

Dark spikes or "hot pixels" and "column blemishes" are the result of single-pixel defects in the SSI CCD array. They are presumed due to high-energy particle radiation damage from solar flares and/or from neutrons generated by the spacecraft's radioisotope thermoelectric generators (RTGs). At a defect site, excess charge is integrated during the time from the end of the CCD erasure preceding an exposure to the beginning of the image readout. As the image is clocked out of the array, each subsequent charge packet in the same column passes through the defect site and collects excess charge, but only during the much shorter single-line readout time. The result is a single pixel (denoted the "head pixel") at a significantly elevated signal level relative to background (perhaps even saturated, depending on gain state and exposure mode), with a single column of pixels, less elevated over background (by a fraction of a DN to a few DNs) and extending from the head pixel to the bottom of the frame, forming a "column blemish.' Depending on a particular dark spike's charge accumulation rate, the frame readout rate, and the gain state, only the head pixel may generate enough signal to be visible in a given image. The characteristic appearance of these blemishes in an actual SSI image is illustrated in Fig. 5 .

Although all head pixel dark spikes were tracked during the interplanetary cruise phase of the mission, it was decided that during the orbital phase only dark spikes intense enough to produce visible column blemishes in the images would be tracked. These were documented on an orbit-byorbit basis and made available for use in image processing software that could attempt to cosmetically remove column blemishes from the relevant images by interpolating across the two columns immediately on either side of the column blemish (Sec. 4).

It was also of interest to assess how individual column blemishes changed with time and to determine how the total number of column blemish dark spikes on the CCD changed with time. Usually only frames in the highest gain state were examined, as any column blemishes visible in lower gain images would almost certainly also be seen in the higher gain frames. Lower gain frames in the slower frame rates were also checked in some orbits, particularly where there were very few or no high-gain frames in the slower frame rates. The types of images examined most often were those with large areas of dark sky or relatively low signal levels and typically fell into the categories of Jovian aurora, Jovian rings, Jovian atmosphere at $889 \mathrm{~nm}$, Io eclipse imaging, small inner satellite full-disk images,

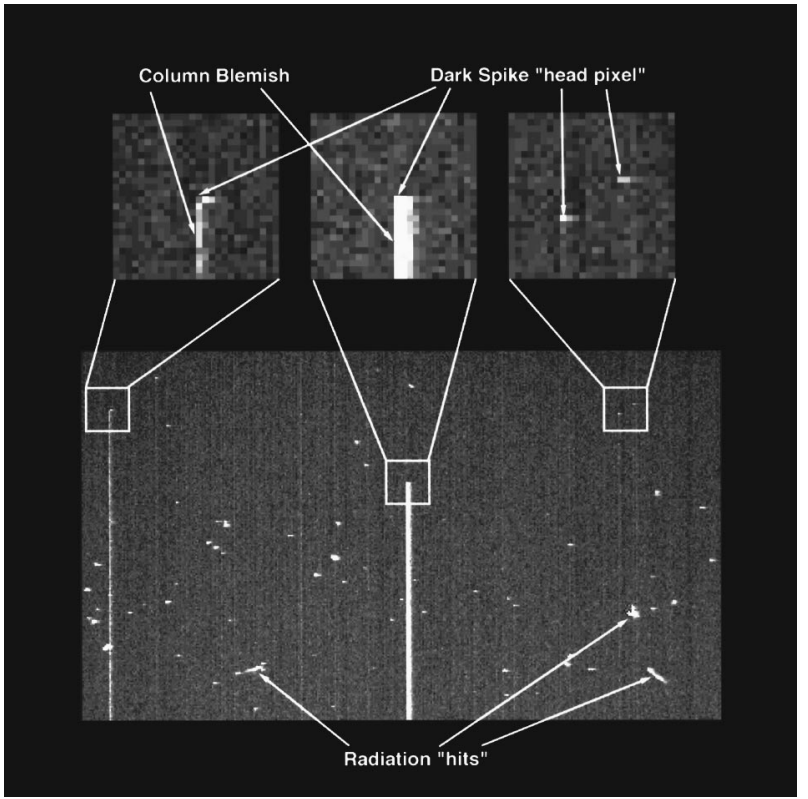

Fig. 5 Portion of an SSI zero-exposure calibration frame taken during the C9 orbit showing examples of dark spikes and column blemishes. Insets show magnified views of the head pixel region for several examples. Some of the column blemish signal is spread over several columns to the right of the blemish due to the CCD charge trap in column 170 (Sec. 2). Irregular clusters of bright pixels in the image are produced by high-energy charged particle interactions (Sec. 6.2).

and distant global observations of the Galilean satellites. Many of these, particularly in the latter three categories, were OCMs.

A number of factors affect the visibility of column blemishes. They are most easily seen in black-sky areas of images that use the slowest frame readout rates and the highest gain states. These conditions provide the greatest contrast between the column defect and its surroundings. Additional factors come into play with the use of partial frame imaging modes, partially recorded frames, and playback cutout-windowing made possible by the new flight software capabilities used to return data from Jupiter orbit. These techniques result in incomplete coverage of the CCD array for the purpose of column blemish inventory. Another important factor is lossy compression of the image data (Sec. 7), which can blur the less intense column blemishes and obscure them with artifacts.

The detectability of column blemishes in the data from a particular orbit is significantly dependent on how these different factors combine for that orbit. For example, orbits E4 and E6 had relatively poor downlink capability, and as a result, compression ratios were relatively high, and heavy use was made of cutout windowing on playback. This reduced the number of column blemishes seen in those orbits. The reverse was true for G8. C10 cruise data included a large amount of black sky and heavy use of the normally seldom used slow HIM readout mode, and the number of column blemishes seen was relatively high. Although the number of column blemishes has varied from orbit to orbit, there has been no statistically significant trend. The selection effects associated with the factors described appear to explain all that has been observed. Based on analysis of all 
Table 5 Number of frames checked for column blemishes, and number of blemishes observed, for each orbit of the nominal mission.

\begin{tabular}{lcc}
\hline \hline Orbit & $\begin{array}{c}\text { Number of Frames } \\
\text { Checked }\end{array}$ & $\begin{array}{c}\text { Number of Column } \\
\text { Blemishes }\end{array}$ \\
\hline G1 & 15 & 7 \\
G2 & 4 & 5 \\
C3 & 17 & 8 \\
E4 & 12 & 6 \\
E6 & 14 & 3 \\
G7 & 25 & 9 \\
G8 & 31 & 14 \\
C9 encounter & 32 & 6 \\
C9 cruise & 5 & 22 \\
C10 encounter & 11 & 12 \\
C10 cruise & 36 & 19 \\
E11 & 10 & 10 \\
\hline \hline
\end{tabular}

visible dark spikes (not just column blemishes) catalogued during interplanetary cruise, ${ }^{3}$ it was expected that the total number would continue to rise gradually through the remainder of the mission; such a continued gradual increase has not been confirmed.

Although the total number of column blemishes is at an approximate steady-state level, individual blemishes did not generally persist over the entire orbital tour. Individual blemishes were typically seen for several orbits (several months) and then disappeared. Few of those seen at the end of the nominal orbital tour were the same ones seen at the beginning. This "annealing', behavior was also observed during interplanetary cruise. ${ }^{3}$ New blemishes have formed at a rate of roughly two to three per orbit.

A comprehensive study of dark spike intensity, as defined by the anomalous signal accumulation rate, has not been carried out. However, spot checks of the rate (calculated by subtracting the mean background DN level in the image from the head pixel $\mathrm{DN}$, converting from $\mathrm{DN}$ to electrons using the appropriate gain state conversion factor, and dividing by the appropriate integration time) show that the intensities are comparable to those seen during interplanetary cruise. In the G8 orbit, for example, the observed column blemishes had charge integration rates of 2000 to 12,000 electrons/s, and in G2, 3300 to 5000 electrons/s; the maximum observed during cruise was 6000 electrons/s. The limit of detectability of column blemishes in a dark HIS high-gain frame is about 300 electrons/s. There is also evidence for changes in intensity of particular column blemishes over time, as was also observed during interplanetary cruise. Column blemishes that seem to have "disappeared" may have only decreased in intensity sufficiently that only the head pixel can still be detected above background.

Table 5 shows the number of frames examined and the number of column blemishes seen in the data for each orbit. Encounter and cruise periods for orbits C9 and C10 are listed separately because of the significant time lapse between these data sets.

\subsection{Radiation Noise}

Besides the possible creation of dark-spike damage sites, energetic particle radiation also causes increased noise levels in the images. Charged particles traversing the CCD undergo ionization energy loss, resulting in the generation of unwanted signal electrons in the CCD. In the case of incident electrons, bremsstrahlung may result in the production of secondary electrons as well. A single radiation "hit" in an image takes the form of a small cluster of pixels with elevated signal levels; if the particle traverses the CCD obliquely, the cluster is elongated into a streak. The average number of pixels affected per incident particle is about four. The time available for the accumulation of radiation noise at any given line of an image lasts from the time of the last CCD erasure prior to the exposure until the readout of the line (except in the 2 1/3-s AI8 imaging mode, which includes no pre-exposure erasure of the CCD). Since the erasure takes place much more rapidly than the image readout, the noise integration time increases linearly from top to bottom within the frame, from about $1 \mathrm{~s}$ at the top to slightly less than the frame cycle time at the bottom, or even to multiples of the frame cycle time in the case of extended exposures and OCMs. As long as the external radiation flux is fairly constant on a time scale of one frame cycle, the radiation noise will also show a top-to-bottom gradient. In the AI8 imaging mode, the integration time is constant over the frame and is equal to the frame cycle time.

Radiation noise has long been expected to be a problem for the SSI in the Jovian system, particularly within the orbit of Europa. The high levels of noise expected at Io encounters due to Jovian electrons led to the addition of the AI8 imaging mode to reduce noise by summing pixels and reading the image off of the $\mathrm{CCD}$ as quickly as possible. This mode is expected to yield adequate image quality at Io based on prelaunch modeling. The prelaunch model for the response of the SSI to radiation noise was tested enroute to Jupiter with a series of zero-exposure frames that were acquired as the spacecraft passed through the Earth's Van Allen belts during December 1992, the second Earth encounter. To within the relatively high uncertainties involved in the analysis of these data, the results seemed to validate the prelaunch model, ${ }^{3}$ but the uncertainties remained high.

Because imaging was canceled during the close perijove pass and Io encounter at Jupiter arrival in December 1995 as a result of the tape recorder sticking anomaly, ${ }^{3}$ a unique opportunity to obtain direct measurements of the effects of Jovian radiation on SSI imaging between 5.9 and $9.2 R_{\mathrm{J}}$ was lost. Measurements made during the orbital tour characterize the response of the camera to magnetospheric radiation over the range of 9.2 to $20 R_{\mathrm{J}}$, but provide little information on what to expect during the close perijove passes and Io encounters that will occur near the end of the Galileo Europa Mission (GEM). Outside of $20 R_{\mathrm{J}}$, the radiation level is too low either to measure accurately in the images or to significantly affect the quality or compressibility of the data.

Measurements of radiation noise were typically made on distant full-disk or intermediate-resolution images of satellites. A variety of imaging modes (including OCMs) and gain states were studied. Rectangular regions of the images 
were selected that contained only black sky, free from obvious scattered light, column blemishes or other extraneous signal sources. These regions were typically about 2500 to 70,000 pixels in size. Preference was given to regions to the left of the column 170 charge trap ${ }^{3}$ and to regions toward the bottom of the frame, where the noise statistics were best. The radiation charge rate was calculated by measuring the mean DN in the region, subtracting the mean background DN, converting DN to electrons using the appropriate gain state conversion factor, and dividing by the mean integration time for the set of lines making up the region. The background contribution was typically measured from the individual images rather than taken from calibration files because scattered light effects could generally not be completely eliminated in images with a planetary body in the frame. The radiation noise was calculated by taking the square root of (the square of the standard deviation of the total DN distribution in the region minus the square of the standard deviation of the background DN distribution in the region), converting to electrons, and dividing by the square root of the mean integration time. These results were compared with the model predictions of radiation charge rate and noise rate at the range from Jupiter at which the image was taken.

In the radiation model, a radiation transfer parameter $J$ is defined by

$J=n / \operatorname{sqrt}(S)$

where $S$ is the radiation charge per pixel, and $n$ is the root mean square (rms) noise due to radiation. ${ }^{1}$ Originally $J$ was set to 34 . Based on the data from the first two orbits, our original model for the radiation noise rate was increased by a factor of 1.6 at $9.4 R_{\mathrm{J}}$ and below transitioning to a factor of 1.4 at $15 R_{\mathrm{J}}$ and above by changing the value of $J$ to 54 below $9.4 R_{\mathrm{J}}$ transitioning to 46 above $15 R_{\mathrm{J}}$. The model for radiation charge rate fit the early data adequately and was not modified.

Although the updated model continued to fit the combined data set well on average for the entire nominal mission, the dispersion of the data about the average was fairly large and became larger toward the end of the mission. Up through orbit G8, the charge rate measurements varied by about a factor of 2 to 3 about the average, but in orbit C9 they were up by a factor of 6 higher thann the average. In the following orbit, C10, a deviation of a factor of about 6 in the opposite direction was observed. The noise also deviated in the same directions but to a lesser degree, as would be expected. The wide dispersion can be seen in Fig. 6 , which is a plot of the measured radiation charge rate as a function of range to Jupiter. In Fig. 7, the $J$ parameter is plotted against the total radiation charge per pixel for the image areas measured. We can see that the measurements agree well with the current model on average. The distribution in $J$ broadens at low radiation charge values due to the difficulty of making statistically meaningful measurements of charge and noise when the number of individual "hits" is relatively small.

The measurements from any one orbit are derived from images taken over several Jovian rotations. They tend to deviate from the long-term average to a similar extent and show no apparent periodicity with time. This suggests that

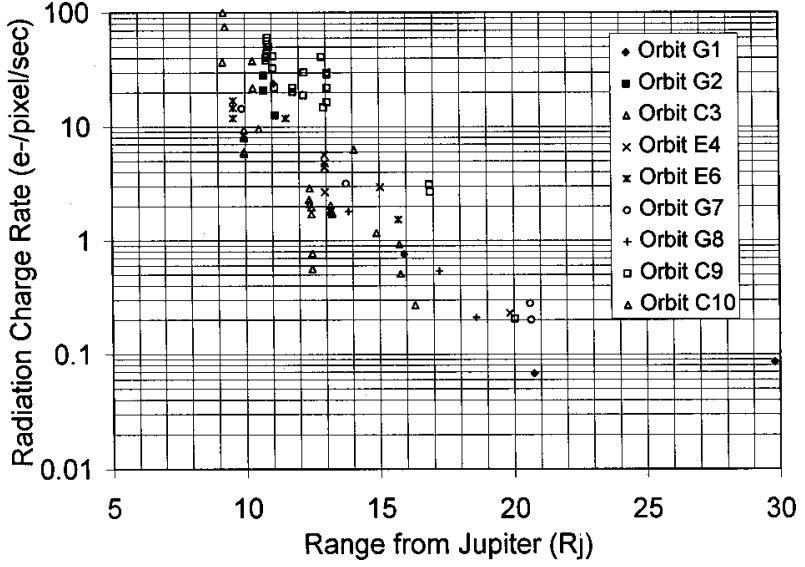

Fig. 6 Radiation-induced signal rates in SSI images as a function of the spacecraft distance from Jupiter in Jovian radii $\left(R_{J}\right.$ $=71,398 \mathrm{~km})$.

the orbit-to-orbit variability is due primarily to long-term (weeks to months) changes in the Jovian magnetosphere as a whole rather than to spatial variations associated with particular Jovian subspacecraft longitudes. The large magnitude and apparent unpredictability of the temporal changes reduce the usefulness of the model in predicting noise levels in individual images, even though it may agree well as a long-term average. Fortunately, for imaging at the orbit of Europa and beyond, even the higher levels of radiation seen at C9 make a relatively small contribution to the total image entropy for most types of scenes (the chief exceptions being Io eclipses and other low-light-level imaging). This, however, makes even more uncertain the expectations for the quality and compressibility of the highresolution Io imaging planned at the conclusion of the extended mission. The nominal expected SNRs for SSI images at Io are estimated to range between 10 and 50, depending on observing geometry, but this range could shift by at least a factor of 2 due to uncertainties in the radiation environment at Io. We see no way to reduce this uncertainty prior to encountering Io.

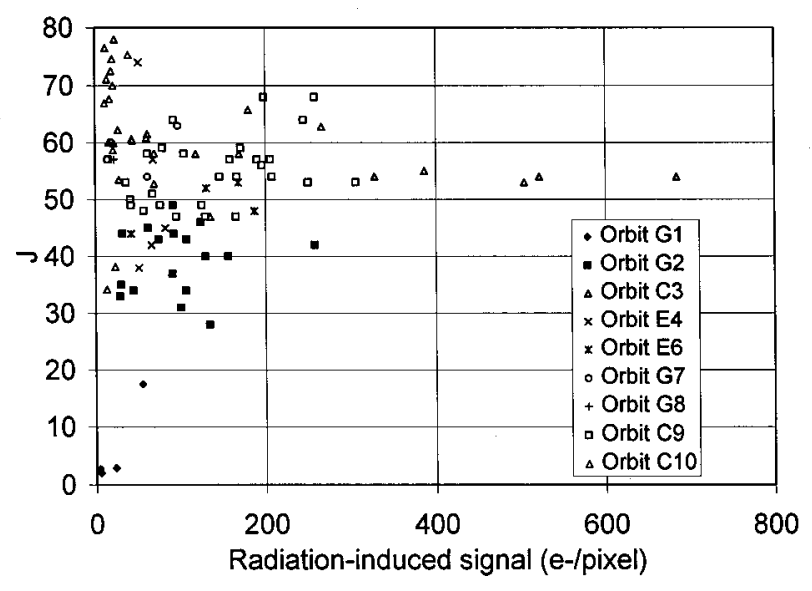

Fig. 7 Relationship between radiation-induced noise and signal in SSI images taken in Jupiter orbit; $J=$ noise/(signal) ${ }^{1 / 2}$. 
Klaasen et al.: Calibration and performance of the Galileo ...

\section{Data Compression Performance}

A new lossy ICT compression algorithm was implemented for orbital operations to mitigate the effects of the reduced downlink capability over the low-gain antenna. ${ }^{3,11,12}$ A new lossless (Huffman) compression capability was also added. In addition, new image editing capabilities were implemented to enable return of selected subareas (cutout windows) of images, as well as small, losslessly compressed "truth windows"' within ICT-compressed frames. An onboard image "despike" capability was made available to reduce the amplitudes of the small bright spots produced in images by energetic particle radiation interactions and thereby to improve data compressor efficiency.

\subsection{ICT Operations and Performance}

The ICT compressor has performed generally as anticipated throughout the orbital mission. Compression ratios are not controlled directly but are achieved by specification of a quantizing factor $Q$ that is applied to the coefficients of the cosine-transformed data. As a result, target compression ratios are not precisely achieved; some images compressed more than predicted, and others compressed less than expected. ICT artifacts, which show up as blocky patterns correlated with the $8 \times 8$-pixel block size on which the compressor works, were visible in many ICT-compressed images; however, in most cases these artifacts did not exceed expected levels.

On rare occasions, the ICT compressor computations encountered a mathematical overflow condition that corrupted the data reconstruction within a particular $8 \times 8$-pixel block. This overflow occurs when extremely high contrast levels are encountered in linear features about 1 pixel wide oriented diagonally within the block. However, this type of data corruption has been observed in only about $208 \times 8$ blocks within the entire orbital mission data set.

Experience using the ICT compressor has led to an improved understanding of the accuracy of the reconstructed data when large quantization factors $Q$ are used. It was intended that the ICT algorithm would preserve, without error, the mean DN value within each $8 \times 8$ block, but that the distribution of individual DN values within the box might change due to the lossy nature of the compression. However, a closer evaluation of the flight algorithm (in reaction to some surprising results in certain images) revealed that the normalization and quantization process that the ICT compressor executes results in the mean value of each block being quantized in steps of $Q / 8$. As a result, we observed images returned with $Q=28$ having reconstructed mean block values separated by 3 -DN steps, $Q=35$ having 4-DN separations, and $Q=255$ having 32-DN separations. The mean value of any ICT-compressed $8 \times 8$-pixel block can therefore be in error by $\pm Q / 16$ DNs. This improved understanding led us to avoid using $Q>24$ beginning with the sixth orbit.

\subsection{Science Impacts of Data Compression}

Target compression ratios were established on an imageby-image basis as a function of the scientific goal of the image, illumination conditions, predictions of SNR and maximum DN, prior experience with the target or type of
Table 6 Typical $Q$ factors and compression ratios for Jovian bodies.

\begin{tabular}{lcc}
\hline \hline Target & Typical $Q$ Factors & Compression Ratios \\
\hline lo & 4 to 8 & $4: 1$ to $14: 1$ \\
Europa & 4 to 10 & $2: 1$ to $5: 1$ \\
Ganymede & 6 to 16 & $2: 1$ to $6: 1$ \\
Callisto & 7 to 11 & $2: 1$ to $5: 1$ \\
Jupiter & 2 to 9 & $5: 1$ to $18: 1$ \\
\hline \hline
\end{tabular}

image (if any), and other factors. In some cases, there was insufficient time to transmit all recorded data at maximum scientifically tolerable compression ratios, resulting in some data being deselected from playback to preserve the scientific fidelity of those data that were returned. Under these conditions, the imaging team was challenged to maximize scientific return by making image-by-image compromises of scientific quality versus quantity. The compression strategy matured progressively throughout the course of the orbital mission as our experience using the compressor increased. Prior to arrival at Jupiter, studies were performed with existing Voyager and Galileo E/M-2 images to establish scientifically acceptable ranges of $Q$ factors and resulting compression ratios for a variety of image targets and types. ${ }^{3}$ Once orbital operations began, the acquisition of images under a range of illumination and resolution conditions gradually established a base of experience for improving the prediction of compression ratios for each target over a range of $Q$ factors. As revealed in the discussions that follow, different approaches were taken by SSI science team members responsible for developing the playback strategy for each target body. The ultimate range of $Q$ factors and resultant compression ratios eventually adopted for each target body during the nominal mission (starting with the sixth orbit) are summarized in Table 6.

Illustrating the complex relationship between $Q$ factor, entropy, compression ratio, and image quality for SSI images of the various Jupiter system bodies is beyond the scope of this paper. Suffice it to say that for the range of compression ratios given in Table 6, compression-related image artifacts would not be apparent to a casual observer.

\subsubsection{Compression of lo images}

Most of the Io images returned during the primary mission have undergone lossy ICT compression. ICT compression works especially well on black space, common in full-disk images, because it provides a high compression ratio with a moderate $Q$ factor, which avoids overly degrading the target body part of the image. The image quality resulting from ICT $Q$ of 5 or less proved satisfactory, but sometimes higher $Q$ factors were used when necessary to return a sufficient number of observations within a limited downlink data allocation. The compression strategy that was adopted for Io was to first calculate the expected downlinked data volume using $Q=5$ on all images and then to make a variety of adjustments (changing $Q$, using cutout windows, deselecting images, or adding Huffman-compressed windows) to increase or decrease the expected data volume and 
optimize the scientific return. The resulting compression ratios vary widely depending on the amount of black sky, noise, and scene contrast.

Jupiter's intense radiation environment is a significant additional complication. The radiation-induced noise in the images increases toward Jupiter and as a function of residence time on the CCD. Range to Io roughly corresponds to range to Jupiter, so the highest resolution Io images tend to be the noisiest. Noisy images are difficult to compress, so they are more expensive in bits returned. This problem is minimized by using the fastest frame times in IM8 (fullresolution) and AI8 ( $2 \times 2$ summation) modes. An additional complication is that ICT compression of bright noise spikes results in "contamination" of other pixels in an 8 $\times 8$-pixel ICT compression block. We used the onboard despike algorithm to minimize this effect, using a threshold signal difference of $50 \mathrm{DNs}$ to detect and remove only the very brightest spikes without changing valid data. In the early orbits, we used a threshold of 30 DNs, but this degraded the bright limb in places. This despiking has a negligible effect on the compression ratio.

OCMs have been used often to acquire several full-disk images (with different colors or exposure times) on a single SSI frame so as to optimize the tape usage. Unfortunately, the use of OCMs increases the radiation-induced noise and lowers the compression ratio at a given $Q$ value. We have nevertheless found this trade-off to be worthwhile when the spacecraft distance to Jupiter exceeds about $15 R_{\mathrm{J}}$.

The few images allocated to Io in each orbit have been carefully planned to achieve the highest priority science results. ${ }^{13}$ The effort to optimize the data return has required us to predict Io's volcanic activity and appearance, often unsuccessfully. Monitoring of active plumes has proven to be especially difficult. We attempted to acquire a global plume inventory in orbit G2 using a set of 31 very highly compressed images $(\sim 30: 1$ compression after extracting cutout windows) designed just to identify probable plumes for better targeted imaging in subsequent orbits. However, only one large bright plume was active at the time, and compression artifacts prohibited identification of small or faint plumes. Nevertheless, during the prime mission, 10 active plumes were imaged and their activity monitored.

Looking ahead to the possibility of close Io flybys at the end of the extended Galileo Europa Mission, the unknown radiation environment at Io's distance presents large uncertainties in expected compressor performance. Fortunately, there is ample downlink after the first Io flyby to return all images at an average compression ratio of just 1.8:1. We plan to return only Huffman-compressed (lossless) images in playback pass 1 through the tape recorder and then evaluate how to compress or select data to return in pass 2 .

\subsubsection{Compression of Europa images}

Prior to arrival at Jupiter, relatively poor Voyager coverage of Europa ( $2 \mathrm{~km} /$ pixel over about $20 \%$ of the surface) provided little help for predicting scientifically tolerable $Q$ s for compression of much higher resolution Galileo images. At the same time and for the same reasons, maximizing the quality and quantity of SSI images of Europa was a high priority for the SSI team, as these images were expected to revolutionize our understanding of the satellite. A further uncertainty involved the amount of image radiation damage to expect near $9.4 R_{\mathrm{J}}$ at the orbit of Europa. Radiation noise increases scene entropy, which requires higher values of $Q$ (causing more degradation to scientific content) to maintain the same compression ratio. Prior to arrival at Jupiter, the amount of radiation and its effect on images were uncertain by factors of 2 to 3 . Previous studies suggested a maximum $Q$ of 8 for general geological interpretation, increasing to as much as 11 or even 16 for some science goals in higher contrast situations. ${ }^{3}$ Actual experience at Europa showed that $Q$ factors of 10 to 13 resulted in little image degradation and typical compression ratios of $2.7: 1$ to $3.8: 1$ for images with resolutions $<250 \mathrm{~m} /$ pixel at incidence angles $>70$ deg. Images with lower incidence angles, lower resolution, and lower intrinsic contrast (e.g., 889-nm and 1- $\mu \mathrm{m}$ filter images) required lower values of $Q$ to meet the scientific goals of the SSI team. When the low relief of Europa's surface and incidence angles below $70 \mathrm{deg}$ reduced scene contrast, $Q$ factors as low as 3 to 5 proved necessary for resolutions $>1 \mathrm{~km} /$ pixel. These experiences resulted in adopting target compression ratios of $3: 1$ to $3.5: 1$ for most Europa images planned during the GEM. Europa has very few recognizable craters, suggesting that its surface may be geologically quite young; therefore, the onboard despiker was not used on Europa images so as to guarantee that the signatures of any small, bright craters at the limit of resolution would not be inadvertently removed or modified.

\subsubsection{Compression of Ganymede images}

One of the many surprises that emerged from Galileo's satellite imaging was the extremely high entropy of Ganymede's surface at high resolution. This is due to its highly tectonized surface and its extremely heterogeneous albedo as expressed at low incidence angles. ${ }^{14}$ This was noted on return of the first images of Galileo's orbital mission, those of Uruk Sulcus, which were BARC compressed. High scene entropy, coupled with the BARC compressor anomaly discussed in Sec. 5.1, contributed to truncation of $\sim 20 \%$ of the samples at the end of each image line in these and other BARC-compressed images. Loss of image samples created difficulty in mosaicking together frames of some of the G1 Ganymede mosaics, as unexpected gaps were produced between horizontally adjacent frames. Later use of BARC compression during the Galileo tour utilized updated predictions of line truncation; frames were positioned with greater degrees of horizontal overlap, overcoming any mosaicking difficulties.

The extreme entropy typically contained in images of Ganymede's surface meant that high $Q$ factors had to be selected if the desired ICT compression ratios were to be achieved for subsequent high-resolution Ganymede imaging. During orbit $\mathrm{G} 2$, for example, $Q=49$ was used in the Nippur Sulcus target region, closely matching the target compression ratio of 8:1. (Some images overcompressed, however, necessitating revisions to predicted $Q$ factors to achieve a desired compression ratio, notably for images obtained in AI8 summation mode.)

In most of the Ganymede images, visible compression artifacts begin to show at an ICT compression ratio of about 3.5:1 to $4: 1$, independent of the $Q$ factor required to achieve this compression ratio. In general, we find that images of Ganymede's grooved terrain, which are of extremely high scene entropy, require a higher $Q$ factor to 
achieve a desired compression ratio than do those of lower entropy dark terrain. Relatively high compression ratios $(\sim 7: 1$ to $8: 1)$ did not greatly affect the interpretability of grooved terrain images. However, such high compression ratios are a hindrance to interpretability of smoother terrains on Ganymede, specifically, smooth regions of bright terrain and dark terrain, in general. Though occasionally distracting, the presence of ICT compression blocks does not severely hurt the interpretability of Ganymede images. Image reprojection, for example, lessens the aesthetic distraction of ICT compression blocks. The level of radiation noise in typical Ganymede images is quite low, and little improvement in image compressibility is achieved by using onboard image despiking.

Occasional image overcompression does hinder interpretation of fine-scale details in some Ganymede image data. Liberal use of truth windows (losslessly compressed 96 $\times 96$-pixel subareas of an image), ${ }^{3,12}$ along with a two-pass tape playback strategy (see Sec. 9) and (on later orbits) return of losslessly compressed cutout windows, helped to overcome occasional problems of overcompression of the Ganymede data. Overall, little or no loss in interpretability of Ganymede images was noted for compression ratios of less than $3.5: 1$ to $4: 1$. Severely tectonized grooved terrain generally retained interpretability to higher compression ratios (up to $7: 1$ to $8: 1$ ); however, dark terrain and smooth regions of grooved terrain lost a significant degree of finescale interpretability at compressions greater than $4: 1$ to $5: 1$. For stereo images, a high compression ratio $(\sim 10: 1)$ for one image of stereo image pairs was found to be entirely suitable for visual interpretation of stereo topography. However, automated stereo matching routines are found to have difficulty in matching points where ICT artifacts are severe, affecting the final resolution of the digital elevation models that can be derived from the image data. ${ }^{15}$

\subsubsection{Compression of Callisto images}

Voyager images of Callisto revealed a heavily cratered surface, and it was expected that impact craters would dominate Galileo images of Callisto even at the highest resolutions. Surprisingly, Galileo images instead revealed a surface that also contains significant areas of dark, smooth material produced by processes of degradation. These dark areas contrast sharply with brighter areas of frost deposits and rough terrain, producing a wide range of scene entropies within individual images. Overall compression ratios no greater than $3: 1$ to $3.5: 1$ were desired to prevent the generation of ICT artifacts within scenes containing abundant low-contrast dark material. The selection of $Q$ and targeted compression ratio depended primarily on resolution, incidence angle, and nature of the target. We used $Q$ factors of 6 to 8 to obtain compression ratios of $3: 1$ to $4: 1$ required for low-resolution images containing bright craters down to the limit of resolution. (In one low-resolution scene, however, a $Q$ factor of 3 yielded a compression ratio of 3.4:1 for a cutout window near the limb.) Moderateresolution $(\sim 800 \mathrm{~m} / \mathrm{pixel})$ images of cratered plains obtained at low incidence angle required higher $Q(\sim 10)$ than those at higher incidence angles $(Q \sim 7)$ to achieve target compression ratios of $3: 1$. However, $500-\mathrm{m} /$ pixel images within the Valhalla multiring system on orbit C9 initially overcompressed by factors ranging from 1.3 to 2.6 com- pared to predicted values due to unexpected low scene entropy. Almost all these data were returned again during a second playback pass with $Q$ ranging from 2 to 5 to achieve an acceptable level of ICT artifacts. High-resolution (35 to $150 \mathrm{~m} / \mathrm{pixel}$ ) image compressibility also varied by target area on Callisto. Only small samples of the first highresolution Callisto data (obtained during orbit C3) were played back on the first playback pass through the tape. Using these samples as a guide, the remainder of the data were successfully played back during the second playback period at compression ratios around 3.5:1 with $Q \sim 8$. Although the average compression ratio of $3.5: 1$ was achieved for these data, smooth areas within the images compressed as high as 6.5:1. This local overcompression resulted in ICT artifacts and loss of small features within some of the images. On $\mathrm{C} 10$, our then-current model of ubiquitous smooth textures on Callisto was modified by two regions imaged at high resolution: the Asgard multiring structure and "smooth plains." These areas turned out to be uniformly knobby in texture rather than smooth, and undercompressed compared to our predictions for the selected $Q$ factor. Limited available downlink enabled BARCcompressed data (six Callisto images) to be returned only on orbit C9 where relatively large amounts of downlink were available; these images showed the frequency of small craters as well as details of the surface texture on Callisto.

\subsubsection{Compression of Jupiter images}

The initial playback strategy for Jupiter observations consisted of ICT compression of whole frames at targeted compression ratios of 10:1 and Huffman compression of smaller cutout windows over features selected from data returned in playback pass 1 . In some orbits, constraints on downlink resources required us to target compression ratios as high as 13:1 (e.g., E6 and G7). In orbit G8, we were able to reduce compression to a targeted value of $6.5: 1$. Standard practice was to keep a 10 to $20 \%$ reserve in our planned downlink usage so that bits would be available in playback pass 2 to fill gaps due to ground station outages during pass 1 and to return Huffman-compressed cutout windows of selected areas.

In orbit $\mathrm{G} 1$, actual compression ratios of $16: 1$ to $20: 1$ were much higher than desired. Significant numbers of blocky, $8 \times 8$-pixel ICT artifacts were visible that confused the tracking of small-scale cloud features. Huffmancompressed subareas over some of these small-scale features were returned in pass 2 to facilitate detailed image analyses. On-board despiking (10 DN threshold) was used to prevent individual spikes from corrupting entire 8 $\times 8$-pixel blocks during ICT compression. Improvements to our planning software better enabled us to predict the amount of compression for given $Q$ factors and scene types, and we became more successful at achieving the desired compression ratios of $10: 1$ to $11: 1$. Experience also taught us that using one $Q$ factor for all frames within an observation caused some frames to overcompress and others to undercompress relative to the desired compression ratiooften substantially so. Allowing $Q$ to vary by frame resulted in more consistent compression ratios, although overcompression and the generation of ICT artifacts remained an issue for some low-contrast scenes taken in the 889-nm methane-2 filter or near the terminator. As a result 
of these improvements in both modeling and strategy, in later orbits Huffman-compressed cutout windows were used much less frequently in pass 2 , particularly as a means to correct for ICT artifacts generated in pass 1. Occasionally, Huffman-compressed cutout windows were returned in pass 2 over interesting small features whose locations were determined based on analyses of the ICT-compressed data returned in pass 1. For example, in orbit E11, Huffman-compressed windows in pass 2 were used to return subsets of images containing possible lightning flashes and the footprint of the Io flux tube that were identified within images returned in pass 1 .

Because of the large number of recorded frames (mostly HIS summation mode), playback efficiency was an important issue for Jupiter observations, more so than for other target bodies. Returning all data in pass 1 and saving pass 2 for Huffman-compressed cutout windows and gap filling was inefficient due to the amount of time spent slewing over deselected parts of the tape in pass 2 . A more efficient strategy required a balanced split between the amount of data returned in each pass. Fortunately, once we were successfully achieving targeted compression ratios of 10:1 to $11: 1$, we were not dependent on using pass 1 to test different $Q$ factors, as was often done for the satellite images. We had the confidence to play back images once, either in pass 1 or pass 2 . Images taken in the near-IR filter were prioritized for return in pass 1 . Pass 2 was used for filling gaps in data sent back in pass 1 as well as returning those images not returned in pass 1 . The disadvantage to this strategy was no ability to fill gaps in data not sent down until pass 2. This proved painful in orbit C9 when bad luck (and bad weather) resulted in more ground station outages in pass 2 than in pass 1 . In general, however, the advantage of improved playback efficiency outweighed a reduced flexibility to fill gaps.

\section{Dependence of Compression Ratio Predictions on the Photometric Models}

Our ability to predict compression ratios progressively improved during the course of the orbital mission. These improvements resulted from updates made to the photometric models of the various scene types imaged (including spectral albedo, albedo contrast, surface slope effects, and spatial frequency effects on scene contrast), the SSI camera response model (including sensitivity and modulation transfer function), the Jupiter radiation model, the relationship of compressibility to image entropy and radiation noise, and elimination of a few bugs uncovered in the SSI planning software.

The improvements in the photometric models ${ }^{16}$ of the target bodies proved to be most important. Some changes to the modeled normal albedo of Jupiter and its satellites were required to make the predicted signal levels match those actually seen in the images. (These changes also improved our ability to select optimum exposure settings.) Table 7 compares the average normal albedo for each filter at the start of orbital operations (top line) to the final values adopted by the end of the primary mission (bottom line, if adjustments were made). Note that the normal albedos listed in Table 7 are appropriate only in combination with the photometric functions used in the SSI software for predicting output signal levels. ${ }^{16}$ For Jupiter, the normal albe- dos relative to model values adopted prelaunch typically increased at high ( $>45 \mathrm{deg}$ ) latitudes and decreased, particularly in the 889-nm strong methane absorption band, in midlatitudes (10 to $45 \mathrm{deg}$ ). Changes rarely exceeded +20 or $-30 \%$. No images were taken of the lit side of Jupiter in the clear, red, or $1-\mu \mathrm{m}$ filters, and only one green image was taken. Therefore, Jupiter albedos in these filters were left unchanged. For the Galilean satellites other than Io (for which the prelaunch albedo models proved adequate), albedos were increased by $15 \%$ for all filters except violet, which increased by $45 \%$ relative to the prelaunch model. The albedo values and photometric functions selected for use at the beginning of the orbital mission matched the available Earth-based and Voyager photometric data well. The updated albedos provide better matches to the Galileo image data obtained. However, the resulting signal level predictions are only accurate to within about $\pm 25 \%$ for the SSI imagery. The reasons for the apparent albedo differences are not known. A more careful and complete photometric analysis is necessary to determine the actual albedos and photometric functions that best fit all of the available measurements.

Major revisions in the modeling of albedo-induced and slope-induced contrast at the limit of resolution were also required to adequately model the image pixel-to-pixel differential entropy, which is the image characteristic most highly correlated to compressibility. Table 8 gives the albedo-induced contrast levels derived from the SSI images that resulted in improved fits to the observed brightness ranges. For comparison, the values used at the beginning of the orbital mission were 0.1 for Jupiter in the visible filters, 0.3 for Jupiter in the near-IR filters, and 0.3 for all the satellites independent of spatial resolution. The revised contrast levels adopted are lower for Jupiter except in the violet filter, for which modeled contrast increased. Contrast was typically increased for the Galilean satellites, particularly at resolutions better than about $1 \mathrm{~km} /$ pixel on Ganymede and Callisto and in the violet filter for all satellites at all resolutions. Europa's contrast in other filters was slightly reduced at resolutions poorer than $200 \mathrm{~m} /$ pixel. These contrasts should be interpreted as typical for each target body.

Table 9 lists the values adopted for the typical surface slopes on the Galilean satellites. At the start of the orbital mission, a value of $10 \mathrm{deg}$ was used for all satellites independent of spatial resolution. Steeper slopes were adopted as resolution increased to better than $2 \mathrm{~km} /$ pixel for all satellites except Europa. For Europa, the model has reduced slopes except at the highest resolution $(<250 \mathrm{~m} / \mathrm{pixel})$ where they are increased.

Another refinement that was made involved permitting the scene contrast to be asymmetric about the predicted mean signal level. The SSI planning software initially modeled the distribution of signal levels due to scene contrasts as being symmetric about the mean. However, for highresolution images of near-terminator scenes, surface slope effects produced highly asymmetric distributions of signal levels. Histograms of DN levels in such images exhibit long tails at high signals due to surface features with sunward facing slopes. Therefore, the modeling software was modified to enable independent calculation of the predicted image mean, minimum, and maximum signal levels. This 
Klaasen et al.: Calibration and performance of the Galileo ...

Table 7 Average normal albedo adjustments made during the orbital mission. Top line indicates albedo used at the start of orbital operations; bottom line indicates albedo adopted by the end of the primary mission.

\begin{tabular}{|c|c|c|c|c|c|c|c|c|}
\hline Target Body & Violet & Green & Clear & Red & Meth 1 & Near IR & Meth 2 & $1 \mu \mathrm{m}$ \\
\hline \multirow[t]{2}{*}{ Jupiter NPH, 70 to $90 \operatorname{deg} N$} & 0.340 & 0.519 & 0.468 & 0.502 & 0.281 & 0.493 & 0.136 & 0.213 \\
\hline & 0.438 & & & & 0.416 & 0.642 & 0.163 & \\
\hline \multirow[t]{2}{*}{ Jupiter NPC, 40 to 70 deg N } & 0.350 & 0.645 & 0.590 & 0.630 & 0.340 & 0.580 & 0.074 & 0.160 \\
\hline & 0.608 & & & & 0.480 & 0.755 & 0.069 & \\
\hline \multirow[t]{2}{*}{ Jupiter NTeB, 32 to $40 \operatorname{deg} N$} & 0.460 & 0.701 & 0.640 & 0.680 & 0.360 & 0.640 & 0.069 & 0.140 \\
\hline & 0.438 & & & & 0.394 & 0.699 & 0.052 & \\
\hline \multirow[t]{2}{*}{ Jupiter NTrZ, 18 to 32 deg N } & 0.586 & 0.787 & 0.670 & 0.713 & 0.394 & 0.681 & 0.075 & 0.149 \\
\hline & 0.499 & & & & 0.351 & 0.751 & 0.053 & \\
\hline \multirow[t]{2}{*}{ Jupiter NEB, 8 to $18 \mathrm{deg} N$} & 0.420 & 0.621 & 0.590 & 0.630 & 0.350 & 0.590 & 0.070 & 0.140 \\
\hline & 0.400 & & & & 0.407 & 0.659 & 0.058 & \\
\hline \multirow[t]{2}{*}{ Jupiter NEqZ, 0 to 8 deg N } & 0.459 & 0.700 & 0.632 & 0.678 & 0.357 & 0.671 & 0.069 & 0.138 \\
\hline & 0.586 & & & & 0.460 & 0.736 & 0.071 & \\
\hline \multirow[t]{2}{*}{ Jupiter Eq, 0 to 7 deg $S$} & 0.380 & 0.700 & 0.631 & 0.681 & 0.370 & 0.620 & 0.080 & 0.171 \\
\hline & 0.609 & & & & 0.476 & 0.768 & 0.078 & \\
\hline \multirow[t]{2}{*}{ Jupiter SEB, 7 to 12 deg S } & 0.400 & 0.592 & 0.531 & 0.573 & 0.312 & 0.523 & 0.067 & 0.143 \\
\hline & 0.544 & & & & 0.382 & 0.681 & 0.062 & \\
\hline \multirow[t]{2}{*}{ Jupiter STrB, 12 to 22 deg S } & 0.420 & 0.621 & 0.590 & 0.630 & 0.350 & 0.590 & 0.070 & 0.140 \\
\hline & 0.470 & & & & 0.399 & 0.722 & 0.042 & \\
\hline \multirow[t]{2}{*}{ Jupiter STrZ, 22 to 26 deg S } & 0.492 & 0.750 & 0.680 & 0.730 & 0.380 & 0.680 & 0.074 & 0.150 \\
\hline & 0.479 & & & & 0.410 & 0.831 & 0.050 & \\
\hline \multirow[t]{2}{*}{ Jupiter STeZ, 26 to 45 deg S } & 0.466 & 0.712 & 0.641 & 0.688 & 0.362 & 0.641 & 0.070 & 0.140 \\
\hline & 0.497 & & & & 0.370 & 0.630 & 0.039 & \\
\hline \multirow[t]{2}{*}{ Jupiter SPC, 45 to $65 \mathrm{deg}$ S } & 0.384 & 0.592 & 0.496 & 0.528 & 0.288 & 0.480 & 0.048 & 0.112 \\
\hline & 0.449 & & & & 0.367 & 0.714 & 0.050 & \\
\hline \multirow[t]{2}{*}{ Jupiter SPH, 65 to 90 deg S } & 0.328 & 0.500 & 0.451 & 0.484 & 0.394 & 0.467 & 0.254 & 0.312 \\
\hline & 0.539 & & & & 0.503 & 0.695 & 0.267 & \\
\hline \multirow[t]{2}{*}{ Jupiter Great Red Spot } & 0.466 & 0.712 & 0.641 & 0.688 & 0.373 & 0.676 & 0.093 & 0.198 \\
\hline & 0.486 & & & & 0.481 & 0.779 & 0.093 & \\
\hline \multirow[t]{2}{*}{ Europa } & 0.496 & 0.750 & 0.776 & 0.790 & 0.780 & 0.780 & 0.760 & 0.750 \\
\hline & 0.719 & 0.863 & 0.892 & 0.909 & 0.897 & 0.897 & 0.874 & 0.863 \\
\hline \multirow[t]{2}{*}{ Ganymede } & 0.295 & 0.420 & 0.450 & 0.460 & 0.460 & 0.450 & 0.440 & 0.430 \\
\hline & 0.428 & 0.483 & 0.518 & 0.529 & 0.529 & 0.518 & 0.506 & 0.495 \\
\hline \multirow[t]{2}{*}{ Callisto } & 0.177 & 0.220 & 0.230 & 0.230 & 0.230 & 0.240 & 0.240 & 0.230 \\
\hline & 0.257 & 0.253 & 0.265 & 0.265 & 0.265 & 0.276 & 0.276 & 0.265 \\
\hline
\end{tabular}

change greatly improved the modeling of high-resolution, near-terminator scenes.

To convert from the peak-to-peak contrast values derived from albedo and slope effects to a statistical measure of the pixel-to-pixel DN differences such contrasts produce, a special parameter is required. We model this relationship as a multiplicative factor $B$ by which the peak-to-peak image contrast is converted to a one-sigma statistical DN difference that applies to adjacent pixels. ${ }^{3}$ Table 10 shows the values of $B$ derived for various targets that generated the best matches to actual image compression ratios. At the beginning of orbital operations, our models used a fixed value of $B=0.28$ for all targets, all filters, and all resolutions. It turned out that the values of $B$ that provided the best compression predictions varied significantly across the range of targets, resolutions, and spectral filters. Because of the lack of Jupiter images in these filters, the green, clear, and red $B \mathrm{~s}$ in the model were set equal to the $727-\mathrm{nm} B \mathrm{~s}$, and the $1-\mu \mathrm{m}$ filter was assigned an extrapolated value of 0.53 for all Jovian latitudes. Determining the best values for $B$ was the greatest challenge and the largest source of error in accurately predicting the compression ratios for the planned images. Perhaps a better model for predicting image entropy than the one we use could be derived for future applications.

Improved compression ratio prediction was obtained by adjusting the modeled SSI MTF at the Nyquist frequency for the $2 \times 2$-pixel summation modes. A lower value for the summation-mode MTF in each filter resulted in better matches to the actual compression ratios. In addition, a refinement to the formula for modeling the effects of image smear on entropy was implemented. The original formula multiplied the inherent scene contrast by the factor 
Klaasen et al.: Calibration and performance of the Galileo ...

Table 8 Albedo-induced contrast levels determined during the orbital mission.

\begin{tabular}{|c|c|c|c|c|c|c|c|c|c|}
\hline Target & Resolution (m/pixel) & Violet & Green & Clear & Red & Meth 1 & Near IR & Meth 2 & $1 \mu \mathrm{m}$ \\
\hline Jupiter & all & 0.20 & 0.08 & - & - & 0.12 & 0.08 & 0.25 & - \\
\hline lo & all & 0.70 & 0.60 & 0.60 & 0.48 & 0.48 & 0.48 & 0.36 & 0.24 \\
\hline Europa & $>610$ & 0.40 & 0.22 & 0.22 & 0.22 & 0.22 & 0.22 & 0.22 & 0.22 \\
\hline Europa & $200-610$ & 0.40 & 0.25 & 0.25 & 0.25 & 0.25 & 0.25 & 0.25 & 0.25 \\
\hline Europa & $<200$ & 0.40 & 0.30 & 0.35 & 0.30 & 0.30 & 0.30 & 0.30 & 0.30 \\
\hline Ganymede & $>1000$ & 0.60 & 0.38 & 0.30 & 0.38 & 0.38 & 0.38 & 0.38 & 0.38 \\
\hline Ganymede & $200-1000$ & 0.60 & 0.60 & 0.60 & 0.60 & 0.60 & 0.60 & 0.60 & 0.60 \\
\hline Ganymede & $<200$ & 1.0 & 1.0 & 1.0 & 1.0 & 1.0 & 1.0 & 1.0 & 1.0 \\
\hline Callisto & $>610$ & 0.40 & 0.40 & 0.40 & 0.40 & 0.40 & 0.40 & 0.40 & 0.40 \\
\hline Callisto & $200-610$ & 0.60 & 0.60 & 0.60 & 0.60 & 0.60 & 0.60 & 0.60 & 0.60 \\
\hline Callisto & $<200$ & 1.0 & 1.0 & 1.0 & 1.0 & 1.0 & 1.0 & 1.0 & 1.0 \\
\hline
\end{tabular}

Table 9 Typical surface slopes (deg) derived during the nominal mission.

\begin{tabular}{lccc}
\hline \hline & \multicolumn{3}{c}{ Resolution } \\
\cline { 2 - 4 } Target & $>2 \mathrm{~km}$ & $250 \mathrm{~m}$ to $2 \mathrm{~km}$ & $<250 \mathrm{~m}$ \\
\hline lo & 10 & 15 & 20 \\
Europa & 5 & 5 & 20 \\
Ganymede & 10 & 15 & 20 \\
Callisto & 10 & 15 & 20 \\
\hline \hline
\end{tabular}

Table 10 Values of $B$ used during the orbital mission.

\begin{tabular}{|c|c|c|c|c|c|c|c|c|c|}
\hline Target & Latitude & Violet & Green & Clear & Red & Meth 1 & Near IR & Meth 2 & $1 \mu \mathrm{m}$ \\
\hline \multirow{15}{*}{ Jupiter } & $\mathrm{NPH}, 70$ to $90 \mathrm{deg}$ & 0.062 & 0.224 & 0.224 & 0.224 & 0.224 & 0.662 & 0.383 & 0.530 \\
\hline & NPC, 40 to 70 deg & 0.069 & 0.330 & 0.330 & 0.330 & 0.330 & 0.662 & 0.383 & 0.530 \\
\hline & NTeB, 32 to $40 \mathrm{deg}$ & 0.110 & 0.205 & 0.205 & 0.205 & 0.205 & 0.280 & 0.130 & 0.530 \\
\hline & NTrZ, 18 to $32 \mathrm{deg}$ & 0.077 & 0.242 & 0.242 & 0.242 & 0.242 & 0.404 & 0.180 & 0.530 \\
\hline & $\mathrm{NEB}, 8$ to $18 \mathrm{deg}$ & 0.058 & 0.130 & 0.130 & 0.130 & 0.130 & 0.260 & 0.156 & 0.530 \\
\hline & NEqZ, 0 to $8 \mathrm{deg}$ & 0.052 & 0.220 & 0.220 & 0.220 & 0.220 & 0.450 & 0.400 & 0.530 \\
\hline & $\mathrm{Eq}, 0$ to $7 \mathrm{deg}$ & 0.065 & 0.199 & 0.199 & 0.199 & 0.199 & 0.404 & 0.900 & 0.530 \\
\hline & SEB, 7 to $12 \mathrm{deg}$ & 0.063 & 0.250 & 0.250 & 0.250 & 0.250 & 0.432 & 0.660 & 0.530 \\
\hline & $\mathrm{STrB}, 12$ to $22 \mathrm{deg}$ & 0.076 & 0.290 & 0.290 & 0.290 & 0.290 & 0.501 & 0.466 & 0.530 \\
\hline & STrZ, 22 to $26 \mathrm{deg}$ & 0.083 & 0.370 & 0.370 & 0.370 & 0.370 & 0.450 & 0.560 & 0.530 \\
\hline & STeZ, 26 to 45 deg & 0.097 & 0.440 & 0.440 & 0.440 & 0.440 & 0.680 & 0.441 & 0.530 \\
\hline & SPC, 45 to $65 \mathrm{deg}$ & 0.089 & 0.380 & 0.380 & 0.380 & 0.380 & 0.670 & 0.500 & 0.530 \\
\hline & $\mathrm{SPH}, 65$ to $90 \mathrm{deg}$ & 0.089 & 0.339 & 0.339 & 0.339 & 0.339 & 0.496 & 0.466 & 0.530 \\
\hline & Great Red Spot & 0.059 & 0.222 & 0.330 & 0.222 & 0.330 & 0.484 & 0.404 & 0.530 \\
\hline & Resolution (m/pixel) & & & & & & & & \\
\hline lo & all & 0.16 & 0.10 & 0.22 & 0.15 & 0.16 & 0.20 & 0.36 & 0.47 \\
\hline Europa & $>610$ & 0.30 & 0.19 & 0.39 & 0.28 & 0.28 & 0.28 & 0.28 & 0.49 \\
\hline Europa & $200-610$ & 0.30 & 0.28 & 0.39 & 0.28 & 0.28 & 0.28 & 0.28 & 0.39 \\
\hline Europa & $<200$ & 0.30 & 0.28 & 0.38 & 0.28 & 0.28 & 0.28 & 0.28 & 0.39 \\
\hline Ganymede & $>1000$ & 0.15 & 0.23 & 0.23 & 0.27 & 0.32 & 0.35 & 0.43 & 0.50 \\
\hline Ganymede & $200-1000$ & 0.25 & 0.28 & 0.23 & 0.28 & 0.32 & 0.40 & 0.43 & 0.45 \\
\hline Ganymede & $<200$ & 0.32 & 0.31 & 0.30 & 0.30 & 0.32 & 0.48 & 0.43 & 0.41 \\
\hline Callisto & $>610$ & 0.28 & 0.35 & 0.40 & 0.40 & 0.40 & 0.35 & 0.52 & 0.51 \\
\hline Callisto & $200-610$ & 0.28 & 0.32 & 0.32 & 0.32 & 0.32 & 0.32 & 0.32 & 0.32 \\
\hline Callisto & $<200$ & 0.21 & 0.21 & 0.21 & 0.21 & 0.21 & 0.21 & 0.21 & 0.21 \\
\hline
\end{tabular}


$\sin (0.5 \pi s) /(0.5 \pi s)$

where $s$ is the smear in pixels, to reflect the contrast reduction due to smear. This factor calculates the reduction in effective MTF due to smear but does not model the effects of smear on image entropy well since its value goes to zero when $s=2$, for example. We replaced this term with the factor

$\left(1+n+f^{2}\right)^{1 / 2} /(1+n+f)$

where $n$ is the integer number of pixels of smear and $f$ is the fractional number of pixels of smear. This formula approximates the reduction in the statistical variation in individual pixel DN levels as smear increases. It gradually approaches zero as the smear increases and yields a more realistic model of the effect of smear on image entropy. This, too, helped improve compression ratio predictions.

Figures $8(\mathrm{a})$ to $8(\mathrm{j})$ illustrate the accuracy of our predicted compression ratios using the current models for various combinations of target body and filter. Predicted compression ratios are typically within about $\pm 25 \%$ of those actually realized. Nevertheless, errors as large as a factor of 2 can occur.

\section{SSI Tape Management and Orbital Operations}

The desire to achieve the original Galileo SSI science goals ${ }^{17}$ with drastically reduced data rates and restricted tape use $^{3}$ required that the targeting, recording, and playback strategy for each image be optimized. As a consequence, sequence design and playback were more complex than simply filling the SSI tape allocation with image data and applying an overall compression ratio that would return all data within the downlink allocation. All SSI data were either BARC compressed as they were recorded to tape or were ICT or Huffman compressed as they were read from tape and transmitted to Earth. The choice between these compression modes affected tape allocation management and was finalized as an integral part of the image sequence design phase, well before each encounter. The SSI allocations of tape and downlink for each target (Jupiter, Io, Europa, Ganymede, Callisto, minor satellites, and rings) changed from orbit to orbit but were not necessarily kept in the same relative proportion. This situation occasionally resulted in tape/downlink allocation imbalances that had to be partly corrected by adjusting the relative proportion of tape efficient BARC-compressed images versus downlinkefficient ICT images. In most circumstances, lossy ICT compression was chosen because it allowed higher compression ratios.

Data return over the low-gain antenna precluded any real-time SSI data return, and the tape recorder became an essential subsystem for capturing, storing, and returning SSI images. The rules for recording data safely given the propensity for the tape to stick to an internal record head evolved as the cause of this sticking became better understood. The project elected to never unwind the portion of the tape containing the location of the first major sticking event, during which the tape drive mechanism spun for 17 $\mathrm{h}$ while the tape remained stuck and unmoving, for fear that this location on the tape had been weakened and could break. This decision left $17 \%$ of the total tape capacity wrapped onto a take-up reel and unusable during orbital operations. For orbit G1, tape-use rules included restrictions on using the highest record rates when recording using "backward" tape motion (the tape has four record tracks; two are recorded moving forward and two are recorded moving backward). Thirty-minute low-speed tape slews were required after each high-rate recording, as were short "unstick" movements in the forward direction before each record or playback activity (to ensure free movement of the tape). By the time of planning for the G2 orbit, these rules were significantly relaxed. The 30-min low-speed slews were replaced by 4-min wait periods on "backward" tracks, and use of the highest record rate was allowed on any track. Starting with E4, tape unstick movements were required only when using the backward tracks.

Strict policies governing tape operations during data playback also evolved during the course of the mission. To reduce operational complexity and workload, initial project policy was to restrict data playback to a single pass of the tape recorder during the long quiet period of each orbit when the spacecraft was far from Jupiter over its dark side. This policy was to be relaxed not earlier than the sixth orbit. However, a desire to obtain the earliest possible return of some of the highest resolution images of Ganymede obtained during the G1 encounter led the project to waive this policy on the first orbit. The high-resolution images were returned on a first playback pass, while the bulk of the rest of the encounter data was returned on a second playback pass. Then, because of an anomaly within the near-IR mapping spectrometer instrument and significant overcompression of other recorded science data, substantial downlink capability remained available after the second pass. The project approved a third and eventually a fourth playback pass to take advantage of this extra capability. Orbit G2 playback was restricted to a single playback pass per the original policy, but, starting with $\mathrm{C} 3$, two or more tape passes were used for every playback period. The project policy to complete the playback of each orbit's data prior to the next orbit's encounter recording period was maintained throughout the mission.

The advantages of multiple playback passes were substantial for SSI. For example, any data lost during the first playback pass (due to outages at the receiving stations, for example) could be filled in during the second pass. Another advantage was that data editing and compression plans could be optimized by previewing small samples of the image data from each observation on the first pass and then optimizing the editing/compression plans for the data on the second pass. This strategy allowed for revisions to the playback plans on the basis of both revised science priorities given what was actually seen in the first pass samples and of actual ICT compression performance. Multiple playback passes provided flexibility to adjust playback plans to match the total downlink capability as it evolved. Some inefficiency was introduced by multiple playback passes since time is lost while skipping over recorded data not selected for playback during a particular tape pass. This inefficiency typically amounted to a few percent of the total capability - a penalty that was judged well worth the advantages.

The SSI Imaging Science Team supported the adaptive playback process by revising its playback plans continu- 


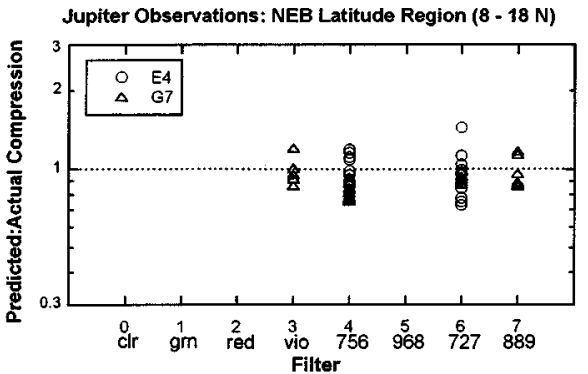

(a)

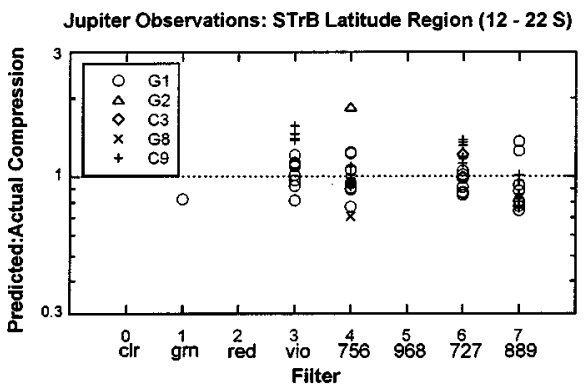

(b)

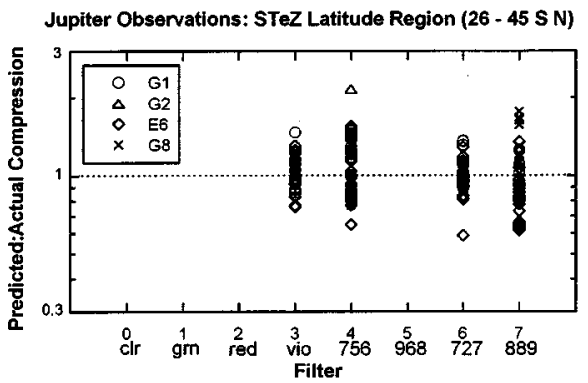

(c)

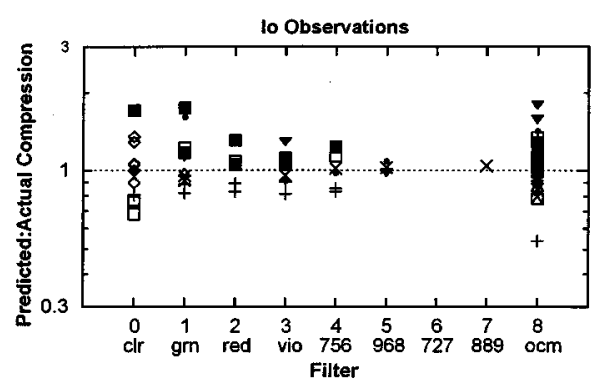

(d)

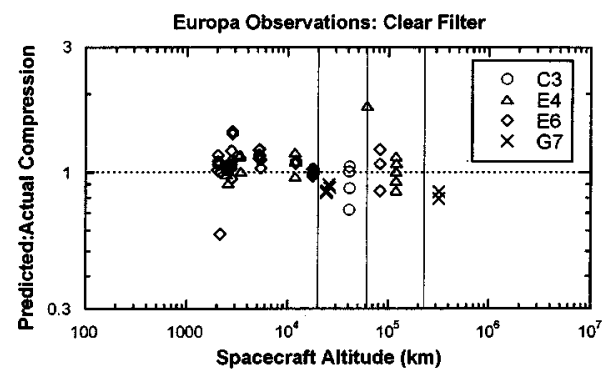

(e)

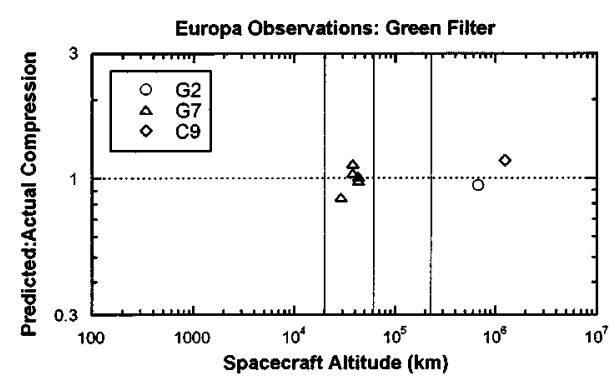

(f)

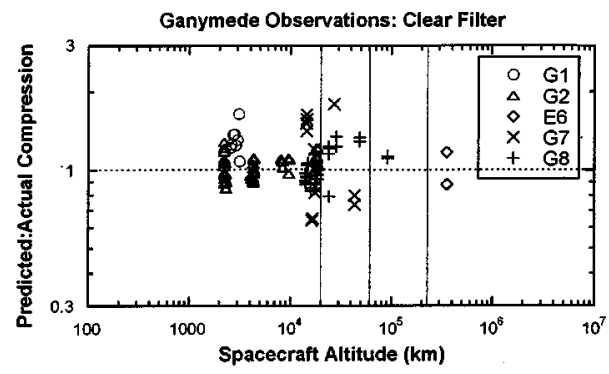

(g)

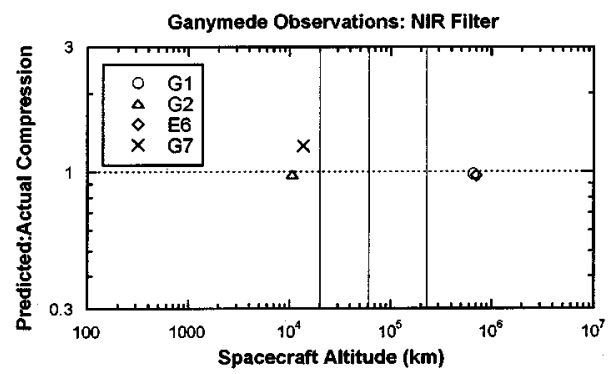

(h)

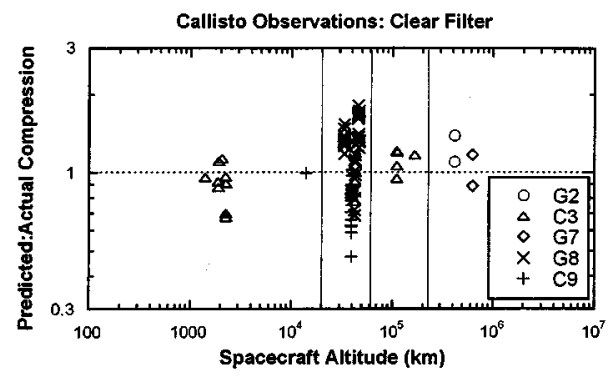

(i)

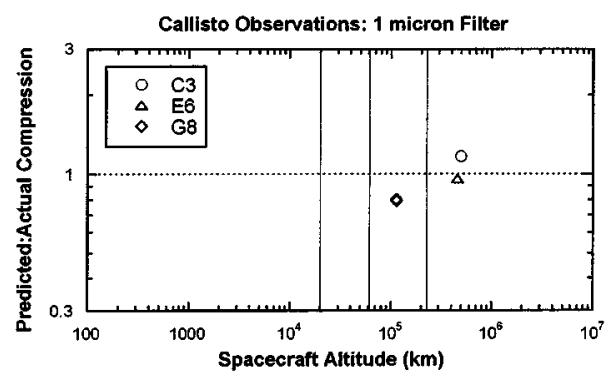

(j)

Fig. 8 Ratios between predicted and actual ICT compression ratios for a variety of SSI images acquired in the Jovian system. Predictions are based on the best-fit models derived by the end of the primary mission. 
Klaasen et al.: Calibration and performance of the Galileo ...

Table 11 Galileo prime mission image return.

\begin{tabular}{|c|c|c|c|c|c|c|}
\hline \multirow[b]{2}{*}{ Target } & \multicolumn{5}{|c|}{ Geometric Resolution (per pixel) } & \multirow[b]{2}{*}{$\begin{array}{c}\text { Total } \\
\text { Frames }^{1}\end{array}$} \\
\hline & $\begin{array}{l}\text { High Resolution } \\
\qquad(<100 \mathrm{~m})\end{array}$ & $\begin{array}{l}\text { Moderate Resolution } \\
(100 \mathrm{~m} \text { to } 1 \mathrm{~km})\end{array}$ & $\begin{array}{l}\text { Regional Coverage } \\
\quad(1 \text { to } 5 \mathrm{~km})\end{array}$ & $\begin{array}{l}\text { Global Mapping } \\
(5 \text { to } 10 \mathrm{~km})\end{array}$ & $\begin{array}{l}\text { Global Mapping } \\
\quad(>10 \mathrm{~km})\end{array}$ & \\
\hline lo & & & 8 & 32 & 169 & 209 \\
\hline Europa & 29 & 48 & 19 & 5 & 34 & 135 \\
\hline Ganymede & 54 & 48 & 10 & 3 & 15 & 130 \\
\hline Callisto & 27 & 69 & 12 & 4 & 11 & 123 \\
\hline Small satellites & & & & 32 & 35 & 67 \\
\hline Rings & & & & & 22 & 22 \\
\hline Total frames for satellite targets & & & & & & 686 \\
\hline \multicolumn{7}{|l|}{ Jupiter } \\
\hline Feature track & & & & & 657 & 657 \\
\hline Lightning/aurorae & & & & & 150 & 150 \\
\hline Total frames for Jupiter targets & & & & & & 807 \\
\hline Total calibration frames & & & & & & 152 \\
\hline Total frames & & & & & & 1645 \\
\hline
\end{tabular}

${ }^{1}$ All unique shuttering events that were played back are counted.

ously as playback took place and images were reconstructed and analyzed. Weekly updates to the playback strategy for the remainder of each orbit's playback period were developed and uplinked to the spacecraft. The SSI team supported these weekly updates through frequent e-mail communication and weekly team teleconferences. During these teleconferences, allocations of downlink capability were made to each science discipline within the SSI team (i.e., each target body) based on review of the science priorities, the data compression results, and evolving total SSI downlink allocations (due to Deep Space Network outages, release of downlink capability from other science teams or from the project-held reserve, or trades with other science teams). Plans for use of the data allocation for each target body were then reviewed by the entire team and approved for implementation.

One new image editing capability was conceived, tested, and put to use during the orbital mission. This capability enabled multiple, vertically separated subareas to be selected for playback from a single image frame. Although this capability was not used frequently, it did prove valuable in certain cases, e.g., to fill in data outages from the first playback pass during the second.

The typical pattern of recording only near each perijove period and playing that data back during the long orbital cruise period of each orbit was occasionally modified. On orbits $\mathrm{C} 9$ and $\mathrm{C} 10$, major periods of additional data recording were scheduled during the cruise periods. On $\mathrm{C} 9$, which was the orbit with the highest apojove and the longest playback period with the most downlink capability, the one and only SSI calibration sequence was scheduled near the time of apojove. These images were recorded on a portion of the tape from which C9 encounter data had already been played back. Some other high-phase-angle Jupiter images were also recorded later in the $\mathrm{C} 9$ cruise period. On $\mathrm{C} 10$, the spacecraft flew through the shadow of Jupiter at a range of about $90 R_{\mathrm{J}}$. SSI took advantage of this opportunity to turn the spacecraft and acquire imagery of the darkside of Jupiter to search for lightning and to observe auroral glows.
These images were also recorded on a portion of tape from which $\mathrm{C} 10$ encounter data had already been played back. These record-during-cruise events required careful planning to ensure that the encounter data to be overwritten were safely returned beforehand.

\section{Data Volume}

Table 11 lists the number of images returned during the Galileo prime mission. Images are tallied for each target in five broad resolution categories that indicate the breakdown between data acquired for detailed, high-resolution mapping, regional coverage and context, and global-scale mapping. Each shuttered frame that was played back wholly or in part is counted. Multiple exposures in different filters in an OCM are counted individually (i.e., not as one image, even though the CCD was read out only once in this type of observation). Images played back more than once during multiple passes through the tape are only counted once. A total of 1645 images was returned during the course of the prime mission. Of the 275 images obtained of Europa, Ganymede and Callisto at better than 1-km/pixel resolution, $110(40 \%)$ are at resolutions better than $100 \mathrm{~m} /$ pixel.

\section{Conclusions}

All SSI science objectives proposed for the low-gain antenna mission have been met or exceeded. Camera performance remained stable and reliable throughout, with little or no change in the instrument calibration from the E/M-2 encounter in 1992 until the end of the mission in 1997. The camera's absolute spectral radiometric response is known to an accuracy of about $5 \%$. The CCD detector remains healthy. Isolated dark spike pixel damage sites continue to form from RTG neutron damage, but many anneal out, and overall performance has not been degraded. The CCD shielding against transient energetic particle radiation interactions performed as predicted, although the Jupiter magnetospheric radiation environment is more variable than expected. 
The availability of a stable, easy-to-use onboard calibration target proved invaluable. Care taken to ensure that the PCT did not become contaminated in flight seems to have been largely successful. The accuracy of some of the SSI calibrations was limited by the inherent measurement limitations of the SSI 8-bit encoding level. Although the SSI optics were well baffled and great care was taken in the development of the camera to minimize scattered light, it turned out to be a significant problem in the scientific analysis of certain types of data. The excellent noise performance of CCD detectors makes the more subtle photometric error sources such as scattered light a greater concern now than was the case with higher noise imagers on previous missions. Measuring the scattered light characteristics completely is a difficult task and probably can be done properly only in space. SSI made a good attempt at this, but much more data and effort would have been required to do the job completely. The ICT data compression and onboard editing capabilities proved invaluable. They provided an added degree of flexibility in observation planning that resulted in returned data of substantially greater value. Predicting data compression ratios for the lossy ICT algorithm proved very challenging. The implementation in flight of new image acquisition and readout modes was essential to the success of the investigation. The multipleexposure OCM capability proved particularly useful. Finally, the use of multiple tape playback passes provided valuable flexibility for recovering lost data packets and optimizing the use of the limited downlink capability.

\section{Acknowledgments}

The success of the Galileo orbital mission is due to the many individual contributions of the flight team and the Deep Space Network under the leadership of W. O'Neil, N. Ausman, R. Mitchell, and T. Johnson. T. Jones planned the SSI observations and playback sequences for the C3 and G7 orbits. Credit goes to the analysts of the JPL Multimission Image Processing Laboratory, including C. Stanley, T. Pauro, A. Culver, D. Jensen, and J. Yoshimizu, for reconstructing and processing the SSI images. A portion of the work described herein was performed at the Jet Propulsion Laboratory, California Institute of Technology, under contract with the National Aeronautics and Space Administration.

\section{References}

1. K. Klaasen, M. Clary, and J. Janesick, "Charge-coupled device television camera for NASA's Galileo mission to Jupiter,' Opt. Eng. 23(3), 334-342 (1984)

2. M. Belton, P. Gierasch, K. P. Klaasen, C. D. Anger, M. H. Carr, C. R Chapman, M. E. Davies, R. Greeley, R. Greenberg, J. W. Head, G. Neukum, C. B. Pilcher, J. Veverka, F. P. Fanale, A. P. Ingersoll, J. B. Pollock, D. Morrison, M. C. Clary, W. Cunningham, and H. Breneman, "Imaging of Venus from Galileo: early results and camera performance," Adv. Space Res. 12(9), 91-103 (1992).

3. K. Klaasen, M. Belton, H. Breneman, A. McEwen, M. Davies, R. Sullivan, C. Chapman, G. Neukum, C. Heffernan, A. Harch, J. Kaufman, W. Merline, L. Gaddis, W. Cunningham, P. Helfenstein, and T.
Colvin, "Inflight performance characteristics, calibration, and utilization of the Galileo SSI camera,"' Opt. Eng. 36(11), 3001-3027 (1997).

4. K. Klaasen, "Galileo solid-state imaging subsystem calibration report: part 2,' JPL Document 1625-210 (1993).

5. A. Cochran and T. Barnes, "Spectrophotometry with a self-scanned silicon photodiode array. I. Instrumentation and reductions,' Astrophys. J., Suppl. Ser. 45, 73 (1981).

6. A. Cochran, "Spectrophotometry with a self-scanned silicon diode array. III. A catalogue of energy distributions,', Pub. Astr. 16, University of Texas (1980).

7. A. Cochran, "Spectrophotometry with a self-scanned silicon photodiode array. II. Secondary standard stars,' Astrophys. J., Suppl. Ser. 45, 83 (1981).

8. H. Breneman and K. Klaasen, "Solid-state imaging subsystem calibration report: part 1,' JPL Document 625-210 (1988).

9. E. Eliason and A. McEwen, "Adaptive box filters for removal of random noise from digital images," Photogramm. Eng. Remote Sens. 56, 453-458 (1990)

10. R. Rice, E. Hilbert, J. Lee, and A. Schlutsmeyer, "Block adaptive rate controlled image data compression,', in Proc. 1979 National Telemetering Conf., Washington, DC (1979).

11. W. O'Neil, N. Ausman, T. Johnson, M. Landano, and J. Marr, "Performing the Galileo Jupiter mission with the low-gain antenna (LGA) and an enroute progress report,' in Proc. 44th Congr. of the International Astronautical Federation, Graz, Austria (1993).

12. K. Cheung and K. Tong, "Proposed data compression scheme for the Galileo S-band contingency mission,', in Proc. of the 1993 Space and Earth Science Data Compression Workshop, pp. 99-109, NASA Conference Publication 3191 (1993).

13. A. S. McEwen, L. Keszthelyi, P. Geissler, D. Simonelli, M. Carr, T. Johnson, K. Klaasen, H. Breneman, T. Jones, J. Kaufman, K. Magee, D. Senske, M. Belton, and G. Schubert, "Active volcanism on Io as seen by Galileo SSI,' Icarus 135, 181-219 (1998).

14. R. T. Pappalardo, J. Head, G. Collins, R. Kirk, G. Neukum, J. Oberst, B. Giese, R. Greeley, C. Chapman, P. Helfenstein, J. Moore, A. McEwen, B. Tufts, D. Senske, H. Breneman, and K. Klaasen, "Grooved terrain on Ganymede: first results from Galileo high-resolution imaging,' Icarus 135, 276-302 (1998).

15. B. Giese, J. Oberst, T. Roatsch, G. Neukum, J. W. Head, and R. T Pappalardo "The local topography of Uruk Sulcus and Galileo Regio obtained from stereo images," Icarus 135, 303-316 (1998).

16. H. Breneman, "Mission sequence system, EXPOSURE calculation subsystem, software requirements document/user's guide,' JPL Document 625-640-211091 Rev. C (1995).

17. M. Belton, K. Klaasen, M. Clary, J. Anderson, C. Anger, M. Carr, C. Chapman, M. Davies, R. Greeley, D. Anderson, L. Bolef, T. Townsend, R. Greenberg, J. Head, G. Neukum, C. Pilcher, J. Veverka, P. Gierasch, F. Fanale, A. Ingersoll, H. Masursky, D. Morrison, and J. Pollack, "The Galileo solid-state imaging experiment," Space Sci. Rev. 60, 413-455 (1992).

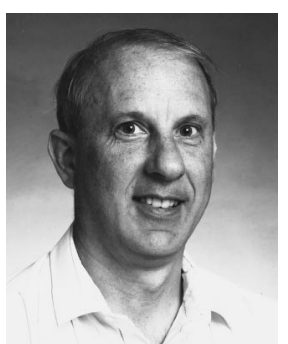

Kenneth P. Klaasen supervises the Imaging Science Group at the Jet Propulsion Laboratory. He received his BS degree in physics from Calvin College and his MS in aerospace engineering from the University of Michigan in 1969. He has been involved in many solar system exploration missions including Mariner 10, Viking Orbiter, and currently the Galileo mission to Jupiter. He served as the experiment representative for the imaging experiments on these missions and is currently a member of the Galileo Imaging Science Team. His primary responsibilities have included imaging system calibration, experiment planning, and mission operations. He has published over 35 papers on the global properties of various planets, satellites, and asteroids and on spacecraft imaging instruments and experiments.

Biographies of the other authors not available. 